\title{
NRF2 Participates in the Suppressive Tumor Immune Microenvironment of KRAS/KEAP1 Co- Mutant Non-Small Cell Lung Cancer by Inhibiting the STING Pathway
}

\section{Xiaodan Sun}

Jilin Cancer Hospital

Peiyan Zhao

Jilin Cancer Hospital

Hui Li

Jilin Cancer Hospital

Yan liu

Jilin Cancer Hospital

Ying Cheng ( $\square$ 18686525037@163.com )

Jilin Cancer Hospital

\section{Research}

Keywords: non-small cell lung cancer, gene co-mutation, tumor immune microenvironment, NRF2

Posted Date: January 3rd, 2022

DOI: https://doi.org/10.21203/rs.3.rs-1175463/v1

License: (c) (1) This work is licensed under a Creative Commons Attribution 4.0 International License.

Read Full License 
$1 \quad$ NRF2 participates in the suppressive tumor immune microenvironment of $K R A S / K E A P 1$ comutant non-small cell lung cancer by inhibiting the STING pathway

3 Running title: Role of NRF2 in KRAS/KEAP1 co-mutant NSCLC

4 Sun Xiaodan ${ }^{1}$, Zhao Peiyan $^{1}$, Li Hui $^{2}$, Liu Yan ${ }^{2}$, Cheng Ying $^{3 *}$

$5 \quad{ }^{1}$ Postdoctoral research workstation, Jilin Cancer Hospital, Changchun, China

$6 \quad 2$ Translational Cancer Research, Jilin Cancer Hospital, Changchun, China

$7 \quad{ }^{3}$ Department of Medical Thoracic Oncology, Jilin Cancer Hospital, Changchun, China

8 * Correspondence: Cheng Ying, chengying@csco.org.cn.

9 Keywords: non-small cell lung cancer, gene co-mutation, tumor immune microenvironment, NRF2

11 Abstract

Background: KRAS/KEAP1 (KK) co-mutant lung adenocarcinoma (LUAD) exhibited poor response to immune checkpoint inhibitors (ICI) via shaping a suppressive tumor immune microenvironment, the mechanism remains to be elucidated.

Methods: The mRNA and protein expression of target molecules were analyzed by qRT-PCR and Western blot, respectively. The subcellular location of NRF2 was observed by immunofluorescence staining, and nuclear and cytoplasm isolation. After exogenous over-expression and knockdown of NRF2 and the addition of a STING pathway inhibitor in tumor cells, the effects on the CD8+ T cell recruitment was detected using chemotaxis assay, and the secretion of chemokines CCL5 and CXCL10 was analyzed by ELISA. The potential NRF2 target BRCA1 was identified using bioinformatic approaches and verified by a dual luciferase reporter assay.

Results: NRF2, the target of KEAP1, was overexpressed and activated in KK type cells. NRF2 effected as a negative regulator of CD8 $+\mathrm{T}$ cells recruitment by decreasing CCL5 and CXCL10 chemokines in KK type LUAD. Mechanistically, NRF2 promoted the transcription and expression of BRCA1 to repair DNA damage, resulting in STING pathway inactivation.

Conclusion: The combination of NRF2 inhibitor or STING agonist with ICI may be a promising therapeutic approach for patients with KK type LUAD. 


\section{Introduction}

29 Lung cancer, which remains the leading cause of cancer-related mortality worldwide, is a major public health concern in the world. ${ }^{1}$ Approximately $85 \%$ cases of histological type are non-small cell lung cancer (NSCLC). ${ }^{2}$ Kirsten Rat Sarcoma $(K R A S)$ is an important oncogenic driver gene of NSCLC and its mutations are found in $20-25 \%$ of NSCLC patients with smoking history. ${ }^{3}$ Although massive efforts have been made to target this protein for decades, little success was achieved due to its special physiochemistry characters, making KRAS an untargetable driver mutation in NSCLC. However, the development of immune checkpoint inhibitors (ICI) brought new hope for patients harboring KRAS mutation. KEYNOTE189, 042 and CheckMate 057 clinical trials had found that NSCLC patients with KRAS mutation could benefit from ICI owing to highly expressed immune checkpoint PD-L1, and increased tumor mutation burden. ${ }^{4-6}$ Interestingly, it should be noted that KRAS mutant NSCLC have distinct co-mutational profiles. Among which, approximately $20 \%$ of $K R A S$ mutation NSCLC underwent loss-of-function mutation of Kelch-like ECH-associated protein 1 (KEAP1), namely the KRAS-KEAP1 co-mutation (KK) type, ${ }^{7}$ which exhibited poorer ICI response compared with individual KRAS mutation $(\mathrm{K})$ type tumors. Therefore, clarifying the molecular mechanism of the poor ICI responsive phenotype of KK type NSCLC is the key to improve the clinical efficacy and break through the therapy bottleneck.

It was reported that co-mutations could regulate the immune contexture to reshape tumor immune microenvironment (TIME), which has been identified as a major influence factor of ICI response. TIME is a complex network which consists of various cell types and factors having important roles regarding tumor development and progression. KK type tumors were confirmed to contain few tumor infiltrating lymphocytes (TILs), especially effect $\mathrm{T}$ cells $\left(\mathrm{CD} 8^{+} \mathrm{T}\right.$ cells), ${ }^{8-10}$ leading to a suppressive TIME; this also can be defined as a cold tumor, ${ }^{11}$ due to the tumor-intrinsic genetic alterations induced the activation of various oncogenic pathways, resulting in the expression of cytokines and chemokines that ultimately mediate the exclusion of $\mathrm{T}$ cells from the TIME, ${ }^{12}$ or, alternatively, the repression of factors that facilitate $\mathrm{T}$ cell recruitment. ${ }^{13}$ In addition, emerging studies has identified stimulator of interferon genes (STING, encoded by TMEM173) pathway as key intracellular pathway bridging anti-cancer innate and adaptive immunity. STING is a cytosolic protein of phagocytic immune, endothelial and cancer cells that gets activated by sensing cytosolic DNA, leads to recruitment and phosphorylation of the kinase Tank Binding Kinase 1(TBK1), which in turn activates the interferon regulatory factor 3 (IRF3) by phosphorylation to produce type I interferons. In addition, it could enhance anti-tumor immune responses via activation of numerous downstream signals, 
which play a major role in recruiting TILs. ${ }^{14}$ Clinical cohort studies from MD Anderson Cancer Center revealed that the expression of STING pathway was associated with immune activation in NSCLC and could be used as markers of identifying NSCLC with an immune-responsive phenotype. ${ }^{15}$ In turn, the tumor cells shaped a suppressive TIME through downregulating STING pathway. ${ }^{16}$

Inactivating mutations in KEAPI lead to activation of its target gene nuclear factor erythroid 2-like 2 $(N R F 2)$, a key transcription factor in cellular antioxidant, metabolic, cyto-protective, and antiinflammatory pathways. NRF2 is well established to be associated with enhanced tumor growth, aggressiveness, and refractoriness to cancer-directed therapy such as radiation, chemotherapy and targeted therapy. ${ }^{17}$ However, no data was available regarding its role in the suppressive TIME of KK type NSCLC. Although a recent study demonstrated that NRF2 was a negative regulator of STING expression, ${ }^{18}$ the underlying molecular mechanisms between them still warrant further study. Thus, this study aims to clarify the role of NRF2 in the suppressive TIME of KK type NSCLC and reveal a novel regulatory mechanism underlying its effect on the STING pathway.

\section{Materials and Methods}

\subsection{Cell culture and Treatment}

The human NSCLC cell lines A549, H460, CALU-1, and H358 were purchased from the Cell Bank of China Academy of Sciences (Shanghai, China), and normal bronchial epithelial HBE cells were obtained from the American Type Culture Collection (VA, USA). A549, H460, and H358 cells were cultured in RPMI-1640 medium (Gibco, MA, USA), CALU-1 cells were cultured in McCOY's 5A medium (Gibco) and HBE cells were cultured in Dulbecco's modified Eagle medium (Gibco). All media were supplemented with $10 \%$ fetal bovine serum (Gibco). The culture environment was $37^{\circ} \mathrm{C}$ and $5 \% \mathrm{CO}_{2}$.

Lipofectamine 3000 reagent (Invitrogen, MA, USA) was used to perform transient transfection according to the manufacturer's instructions. Human NRF2 expression vector and empty vector (GeneChem, Shanghai, China) were used for NRF2 overexpression and negative control, respectively. For knockdown experiments, cells were transfected with NRF2-/BRCA1- specific siRNA or scrambled control siRNA (Genecreate, Wuhan, China) for $48 \mathrm{~h}$ or $72 \mathrm{~h}$.

To inhibit STING signaling, cells were treated with the STING inhibitor H151 (SelleckChem, TX, USA) in DMSO (Sigma-Aldrich, Germany). Cells were treated with different concentrations (0.25 
$\mu \mathrm{M}, 0.5 \mu \mathrm{M}, 1.0 \mu \mathrm{M}$, and $2.5 \mu \mathrm{M})$ of $\mathrm{H} 151$ after transfection and incubated for individual time points

91 of $1 \mathrm{~h}, 3 \mathrm{~h}$, and $5 \mathrm{~h}$.

92

93

\subsection{Immunofluorescence Staining}

A549 and CALU-1 cells were seeded in $20 \mathrm{~mm}$ culture plates, washed with PBS, fixed with 4\% paraformaldehyde for $15 \mathrm{~min}$, and permeabilized in $0.1 \%$ Triton X-100 for 5 min. After blocking with $5 \%$ bovine serum albumin (BSA) for $1 \mathrm{~h}$ at room temperature, the cells were incubated with primary antibody against NRF2 (dilution 1: 100) over night at $4^{\circ} \mathrm{C}$. Then, FITC-conjugated secondary antibody (dilution 1: 200) was incubated with the cells for $1 \mathrm{~h}$ in the dark at room temperature, and the cells were stained with 4',6-diamidino-2-phenylindole for 5 min to visualize the nuclei. Images were captured using a fluorescence microscope.

\subsection{Quantitative Real-time Polymerase chain reaction (qRT-PCR)}

TRIzol (Invitrogen) was used to extract total RNA from NSCLC cells. Reverse transcription was performed using PrimeScript RT Master Mix (Takara, Otsu, Japan). qRT-PCR was performed using SYBR qPCR Mix (TOYOBO, Shanghai, China) on a Real-Time PCR System (MX3000P, Agilent Technologies, CA, USA). The thermocycling conditions: 30 seconds at $95^{\circ} \mathrm{C}$, then 40 cycles at $95^{\circ} \mathrm{C}$ for 5 seconds and $65^{\circ} \mathrm{C}$ for 30 seconds. The mRNA ratio of the target genes to GAPDH was calculated using the $2^{-\Delta \Delta \mathrm{Ct}}$ formula. The primer sequences are shown in Supplement Table1.

\subsection{Western blot}

Total proteins from NSCLC cells were lysed in RIPA (Beyotime, Shanghai, China) lysis buffer supplemented with $1 \%$ phenylmethanesulfonyl fluoride. $40 \mu \mathrm{g}$ proteins were separated using $10 \%$ SDS-PAGE, transferred onto $0.45 \mu \mathrm{m}$ polyvinylidene fluoride membranes (Millipore, MA, USA), and incubated overnight at $4{ }^{\circ} \mathrm{C}$ with primary antibodies (Supplement Table 2), followed by incubating with a horseradish peroxidase-conjugated anti-rabbit IgG secondary antibody. ImageJ software was used to evaluate the gray value of each band.

\subsection{Nuclear and Cytoplasm Isolation}

The Nuclear and Cytoplasmic Protein Extraction kit (Beyotime) was used to isolate the nuclear and cytoplasm cell fractions. The collected cells were suspended in ice-cold hypotonic buffer and incubated on ice for $20 \mathrm{~min}$. The extracts were then centrifuged at $12,000 \times \mathrm{g}$ for $5 \mathrm{~min}$, and the supernatants were collected as cytosolic fractions. The pellets were washed with ice-cold PBS and 
resuspended in lysis buffer, followed by vortexing at the highest speed for $30 \mathrm{~min}$. These extracts were centrifuged at $12,000 \times \mathrm{g}$ for $10 \mathrm{~min}$, and the supernatants were collected as the nuclear fractions.

\subsection{Enzyme-linked Immunosorbent assay (ELISA)}

To evaluate the concentrations of CCL5 and CXCL10 in the cell culture supernatant after different treatments, the ELISA kits (human CCL5/ RANTES ELISA Kit and human CXCL10/IP-10 ELISA Kit, Multi sciences, Hangzhou, China) were used, according to the instruction booklets supplied by the manufacturers.

\subsection{Chemotaxis assay}

Peripheral blood mononuclear cells were isolated from peripheral blood of healthy volunteers using Ficoll-Paque (GE Healthcare, CA, USA) according to the instructions. Transwell chambers $(5 \mu \mathrm{m}$ pore size, Corning, NY, USA) were used to perform the chemotaxis assay. $2 \times 10^{5}$ cells were loaded onto top chamber and cell free supernatant after different treatment was added to the lower chamber. The cells were allowed to migrate for $8 \mathrm{~h}$ at $37^{\circ} \mathrm{C}$. Then the migrated cells were harvested and resuspended in PBS containing 0.5\% BSA and tested with FACS Canto II cytometer (BD, NJ, USA) using antihuman-CD8-FITC (BD) and antihuman-CD3-APC (BD), to determine the percentage of $\mathrm{CD}^{+} / \mathrm{CD}^{+} \mathrm{T}$ cells in the migrated cells and subsequent analysis was performed using FlowJo software.

\subsection{Dual-Luciferase reporter assay}

CALU-1 and H358 cells were seeded in six-well plates at a density of $2 \times 10^{5}$ cells/well and cultured for $24 \mathrm{~h}$. Cells were then co-transfected with BRCA1 promoter/luciferase construct vectors (pGL3BRCA1, control vector pGL3-Basic, GeneChem), and Nrf2 expression vector or empty vector, respectively, together with control Renilla luciferase reporter construct vector (pRL-TK, GeneChem). The luciferase activity was measured using a luciferase assay kit (Promega, WI, USA) according to the manufacturer instructions.

\subsection{Online Database}

A series of online databases were implemented as following: 


\begin{tabular}{|c|c|c|}
\hline Name & Website & Application \\
\hline CCLE & https://sites.broadinstitute.org/ccle & $\begin{array}{l}\text { To identify the mutation type of NSCLC cell lines used in } \\
\text { the study. }\end{array}$ \\
\hline GEPIA & http://gepia.cancer-pku.cn/ & $\begin{array}{l}\text { To compare the mRNA levels of STING in normal and } \\
\text { tumor tissues, and analyze the correlation between NRF2 } \\
\text { and STING or chemokines. }\end{array}$ \\
\hline HPA & https://www.proteinatlas.org/ & $\begin{array}{l}\text { To compare the protein expressions of STING in normal } \\
\text { and lung cancer tissues. }\end{array}$ \\
\hline $\begin{array}{l}\text { Kaplan- } \\
\text { Meier Plotter }\end{array}$ & http://www.kmplot.com/ & To evaluate the prognostic value of STING. \\
\hline TISIDB & http://cis.hku.hk/TISIDB/ & $\begin{array}{l}\text { To analyze the correlation between NRF } 2 \text { and } \mathrm{CD}^{+} \mathrm{T} \text { cell } \\
\text { abundance. }\end{array}$ \\
\hline NCBI & https://pubmed.ncbi.nlm.nih.gov/ & To obtain the promoter sequences of $B R C A 1$ gene. \\
\hline String & https://string-db.org/ & To predict functional associations between proteins. \\
\hline JASPAR & http://jaspar.genereg.net/ & $\begin{array}{l}\text { To predict the binding sequence of } B R C A 1 \text { promoter with } \\
\text { NRF } 2 \text {. }\end{array}$ \\
\hline
\end{tabular}

\subsection{Statistical Analysis}

All experiments were repeated at least three times. Data were analyzed using GraphPad Prism 8.0 software and presented as mean \pm standard deviation. Student's $t$-test and one-way ANOVA were used to compare the differences between two groups or multiple-groups, respectively. $P<0.05$ was considered statistically significant.

\section{Results}

\subsection{NRF2 was overexpressed and activated in $K R A S-K E A P 1$ co-mutation cells}

First of all, we identified the A549 and $\mathrm{H} 460$ cell lines as harboring KRAS-KEAPl (KK) co-mutation type, and the CALU-1 and H358 cell lines as harboring KRAS (K) mutation type through CCLE database (Figure 1A, Supplemental Table 3). Since NRF2 is the direct target gene of KEAP1, mutation of KEAP1 would lead to overexpression and activation of NRF2, we observed the expression of NRF2 in these types of cells through western blot and qRT-PCR. As expected, both the protein and mRNA expressions of NRF2 were higher in the KK type cells than those in the $\mathrm{K}$ type cells (Figures 1B, C). Furthermore, since the activated status of NRF2 is mainly located in the nucleus, 17 the nuclear and cytoplasm isolation assay was performed to observe the subcellular location of NRF2. The results showed that in the KK types cells, NRF2 was mainly in the nucleus 
while located in the cytoplasm of the K type cells (Figure 1D). The cell immunofluorescence staining confirmed these findings as well (Figure 1E). Collectively, these results identified that NRF2 was overexpressed and activated in KK type cells, suggesting a potential role of NRF2 in KK type tumor's development.

\subsection{NRF2 negatively regulated $\mathrm{CD8}^{+} \mathrm{T}$ cells recruitment and its chemokines}

Since KK type NSCLC was reported to have a poor response to ICI through modeling a suppressive TIME, which is charactered as lacking of TILs, especially $\mathrm{CD}^{+} \mathrm{T}$ cell infiltration, we wondered whether NRF2 contributed to the low $\mathrm{CD}^{+} \mathrm{T}$ cell abundance. Firstly, the bioinformatic analysis results from TISIDB database showed that the expression of NRF2 was negatively correlated with $\mathrm{CD}^{+} \mathrm{T}$ cell abundance in 517 cases of NSCLC ( $P=0.0472$, Figure 2A). Besides, CCL5 and CXCL10 are two important and potent chemokines for recruiting $\mathrm{CD}^{+} \mathrm{T}$ cells into TIME, ${ }^{19}$ we observed that the mRNA level of NRF2 was associated with decreased mRNA expressions of these chemokines in NSCLC through GEPIA database (Figures 2B, C). Then, we verified these observations by up-and down-regulating NRF2 in K type cells and KK type cells, respectively. Overexpression of NRF2 attenuated $\mathrm{CD}^{+} / \mathrm{CD}^{+} \mathrm{T}$ cells recruitment (Figures 2D, E, S1A), whereas the opposite effects were observed in NRF2 knockdown groups (Figures 2F, G, S1B). Furthermore, we investigated the effect of NRF2 on the expression and secretory of $\mathrm{CD}^{+} \mathrm{T}$ cells' chemokines. Upregulation of NRF2 resulted in decreasing mRNA levels of CCL5 and CXCL10 (Figure 2H). These effects were reversed by NRF2 knockdown (Figure 2I). Then, the secreted levels of these chemokines in the cell culture medium were tested by ELISA. Consistent with the above findings, overexpression of NRF2 inhibited the secretion of CCL5 and CXCL10 (Figure 2J), whereas NRF2 downregulation had the opposite effects (Figures 2K, S1C). These results indicated that NRF2 could negatively regulate $\mathrm{CD}^{+} \mathrm{T}$ cells recruitment and its chemokines.

\subsection{The tumor suppressor role of STING in NSCLC}

As emerging studies have shown that activation of STING pathway is able to boost $\mathrm{CD}^{+} \mathrm{T}$ cell recruitment by upregulating CCL5 and CXCL10, ${ }^{20,21}$ we speculated that STING pathway may be related with NRF2. As the key protein in this pathway, we briefly studied the role of STING in NSCLC mainly by bioinformatic analysis. Firstly, the mRNA expression of STING was analyzed in a number of human tumors and corresponding normal tissues by GEPIA database. The results showed that in most solid tumors, including NSCLC, the expression of STING was significantly lower in tumor than that in the normal tissues (Figures 3A, B). In addition, the low-level protein 
expression of STING in tumor was confirmed by immunohistochemistry results from the HPA database (Figures 3C). To verified these findings, we evaluated the expression of STING in normal bronchial epithelial HBE cells and two NSCLC cell lines through western blot, of which HBE cells had the highest level of STING (Figures 3D). Furthermore, the results from the Kaplan-Meier Plotter database showed that the low-level expression of STING was associated with better overall survival and progress free survival, suggesting a favorable prognostic value of STING (Figures 3E, F). Taken together, we identified a tumor suppressor role of STING in NSCLC.

\subsection{STING pathway was negatively regulated by NRF2}

To analyze the relationship between STING and NRF2, we searched the GEPIA database firstly. The results suggested a significant negative correlation between them (Figure 4A). Then, the increasing protein and mRNA expressions of STING in the KK type cells compared to the $\mathrm{K}$ type cells were observed (Figures 4B, C), which was just opposite to the expressions of NRF2 (Figures 1B, C). Hence, we supposed that NRF2 may be a negative regulator of STING. To confirm our hypothesis, NRF2 was knocked down in KK type cells using three specific NRF2 target siRNA sequences, of which No.3 siRNA sequence exerted the highest transfection efficiency (Figure 4D), thus, it was selected for subsequent study. Besides, NRF2 was upregulated by transfecting with a constructed NRF2 expression vector in K type cells. The results showed that the expressions of TBK1 and IRF3, which are key target molecules of STING, ${ }^{22}$ remained unchanged regardless of NRF2 levels (Figure 4E). However, the variational expressions of NRF2 affected the expressions of STING, phosphoTBK1 (p-TBK1), and p-IRF3, indicating an increasing activity of STING pathway (Figures 4E, F). These results revealed that STING pathway was negatively regulated by NRF2.

\subsection{STING pathway was crucial for NRF2-mediated suppressive TIME of KK type NSCLC}

To further investigated whether NRF2-medidated suppressive TIME of KK type NSCLC was dependent on STING pathway, rescue experiments were performed in KK type cells, in which NRF2 exhibited high-level expression. As downregulation of NRF2 was proved to stimulate the expression of STING, H151, a chemical compound specific inhibits STING, ${ }^{23,24}$ was applied after knocking down of NRF2 in KK type cells. Based on recent studies about H151, we explored its inhibitory efficacy by a range of concentrations and treatment for different time points. The results showed that the activity of STING pathway was lowest after $3 \mathrm{~h}$ of exposure to $0.5 \mu \mathrm{M} \mathrm{H} 151$ and increased again by $5 \mathrm{~h}$ (Figure $5 \mathrm{~A}$ ), hence, this treatment condition was selected for the subsequent experiments. As proved above, the mRNA expression and secretory level of CCL5 and CXCL10 were both increased 
in response to downregulation of NRF2. However, the application of H151 reversed these effects (Figures $5 \mathrm{~B}, \mathrm{C}$ ). In addition, similar rescue effect of $\mathrm{H} 151$ on the abundance of $\mathrm{CD}^{+} / \mathrm{CD}^{+} \mathrm{T}$ cells was observed as well (Figure 5D). Overall, these results revealed that STING pathway was crucial for NRF2-mediated suppressive TIME of KK type NSCLC.

\subsection{NRF2 promoted BRCA1 expression and DNA damage repair to inhibit STING pathway}

230 Next, the mechanism by which NRF2 suppressed the STING pathway was further investigated. Using the String database, we first excluded the possibility of direct interaction between these two proteins (Figure 6A), suggesting the presence of intermediate mediators. It has been reported that DNA damage is a direct activator of STING pathway, ${ }^{20}$ and BRCA1 is identified as an important DNA damage repair gene, we hypothesized that NRF2 inhibited STING pathway by promoting the expression of BRCA1 and reducing DNA damage. The correlation analysis from GEPIA database confirmed that the mRNA level of BRCA1 was negatively associated with STING whereas positively related with NRF2 (Figure 6B). The protein expression of BRCA1 was higher in the KK type cells than that in the K type cells, which was similar to the expression of NRF2 and opposite to the expression of STING in these cells (Figures 6C, 1B, 4B). Furthermore, overexpression of NRF2 resulted in upregulation of BRCA1, whereas decreased the level of DNA damage marker $\gamma$-H2AX. The opposite effects were observed upon NRF2 knockdown (Figure 6D). These findings raised the question of how NRF2 upregulated BRCA1 expression. Since NRF2 is also functioned as a transcription factor, we wondered whether NRF2 could bind to the promoter of BRCA1. The sequences of antioxidant response element (ARE) are known to be an important binding region of NRF2. It was predicted the presence of the same sequences as ARE in the upstream of BRCA1 gene transcriptional start site based on JASPAR database (Figure 6E). A dual-luciferase reporter assay was performed to verify this conjecture. As expected, we found that when NRF2 was over-expressed, the cells transfected with pGL3-BRCA1 exhibited a clearly increase luciferase activity compared to the pGL3-Basic transfected cells (Figure 6F). Then, we knocked down the expression of BRCA1 through transfecting three specific BRCA1 target siRNA sequences in the KK type cells to study whether BRCA1 mediated the inhibitory effect of NRF2 on STNIG pathway, and No.1 siRNA sequence was selected for it exerted the highest transfection efficiency (Figure 6G). Interestingly, down-regulation of BRCA1 exerted no obvious effect on the levels of NRF2, implying NRF2 may function as an upstream factor of BRCA1. However, the inactivated STING pathway in KK type cells was re255 activated in response to BRCA1 knockdown (Figure 6H). Based on these findings, it was inferred 
that NRF2 inhibited STING pathway by promoting transcription and expression of BRCA1, resulting

257 in reduction of DNA damage.

\section{Discussion}

Compared with $\mathrm{K}$ type tumors, KK type NSCLC exhibited poorer response to ICI via shaping a suppressive TIME, the mechanism of which remains to be elucidated. Here, we demonstrated that NRF2 effected as a negative regulator of $\mathrm{CD}^{+} \mathrm{T}$ cells recruitment by decreasing CCL5 and CXCL10 chemokines. Mechanistically, NRF2 participated in the suppressive TIME of KK type NSCLC by promoting transcription and expression of BRCA1 to repair DNA damage, resulting in STING pathway inactivation (Figure 7).

$K R A S$-mutated NSCLC represents a genetically heterogeneous subgroup with a high frequency of cooccurring mutations in cancer-associated pathways. A recent analysis of 1,078 KRAS-mutant NSCLC showed that $53.5 \%$ of patients harbored at least one additional genomic alteration. The most common mutations were tumor protein p53 (TP53, 39\%), serine/threonine kinase 11 (STK11, 20\%), and KEAP1 (13\%). ${ }^{25}$ This diversity might be involved in the distinct biological behavior of these subgroups. KRAS-TP53 co-mutant tumors were characterized as a hot tumor owing to its immunerich microenvironment with TILs, ${ }^{26}$ whereas, the KRAS-STK11 and KK type tumors were regarded as cold tumors for shaping a suppressive TIME. Most insights were focus on the KRAS-TP53 and $K R A S-S T K 11$, which had higher mutation rates, while the knowledge regarding KK type tumors is still limited. A recent datamining analysis reported that KEAP1/NRF2-mutant lung cancer presented a deficiency of TILs due to substantial downregulation of immunologically related genes. ${ }^{8}$ Here, we found that NRF2 was overexpressed and activated in KK type cells, suggesting a potential role of NRF2 in KK type tumor's development. Through regulating the expression of NRF2 in both K and KK type cells, we observed that NRF2 could reduce the recruitment of $\mathrm{CD} 8^{+} \mathrm{T}$ cells, and negatively regulate the expression and secretion of chemokines CCL5 and CXCL10.

Emerging attention has been drawn on the role of the STING pathway in the TIME. The expression of STING pathway could be used as markers of an immune-responsive phenotype ${ }^{15}$ while its inactivation in tumor represents a suppressive TIME. ${ }^{16}$ In breast cancer and small cell lung cancer, STING pathway activation could induce the expression of CCL5 and CXCL10 and increase the number of $\mathrm{CD}^{+} \mathrm{T}$ cells. ${ }^{20,21}$ Besides, suppression of STING in melanoma and prostate cancer cells led to reduced TILs. ${ }^{27,28}$ Therefore, the STING pathway plays an important role in TIME. Since NRF2 was found to inhibit these chemokines, raising our interested to the relationship between 
NRF2 and STING pathway. We briefly identified a potential tumor suppressor role of STING protein in NSCLC by online database and experimental confirmation. In addition, we revealed that NRF2 was a negative regulator of the STING pathway and its role in suppressive TIME of KK type NSCLC was dependent on its regulation of STING pathway.

It has been reported that NRF2 regulated STING expression in human cells by decreasing STING mRNA stability. ${ }^{18}$ However, the molecular mechanism between them needs further exploration. First, the direct interaction between them was excluded by String database, indicating the presence of intermediate mediators between them. As mentioned before, STING gets activated by cytosolic DNA, including tumor DNAs. It was reported that carboplatin could induce DNA damage to activate the STING pathway, ${ }^{29}$ and targeting DNA damage response protein therapy exhibited enhanced antitumor immunity through STING-mediated T-cell activation in small cell lung cancer. ${ }^{21} B R C A 1$ is known as an important DNA damage repair gene, and based on the predict results from JASPAR database, we identified BRCA1 as the target of NRF2. In addition, we demonstrated that NRF2 promoted the transcription and expression of BRCA1 to reduce DNA damage, leading to suppression of STING pathway.

Altogether, we clarified the effects of NRF2 in the suppressive TIME of KK type NSCLC and revealed a novel regulatory mechanism underlying its effect on the STING pathway. Unfortunately, there is a lack of in vivo experiments to confirm our observations in vitro due to the limitation of our objective conditions. In addition, we failed to clarify the specific domain of BRCA1 to which NRF2 bound. Moreover, since the BRCA1 is not a major DNA damage repair protein in NSCLC, there are still some other molecules mediate the effect of NRF2 on the STING pathway, and the effects of NRF2 on other immune cells are not studied here. Thus, further studies are warranted. Collectively, our study reveals a novel role of NRF2 in TIME and provides a new perspective for ICI treatment of KK type NSCLC. The combination of NRF2 inhibitor or STING agonist with ICI may be a promising therapeutic approach for patients with KK type NSCLC.

\section{Abbreviations}

ARE: antioxidant response element; BSA:bovine serum albumin; ELISA: enzyme-linked immunosorbent assay; ICI: immune checkpoint inhibitors;IRF3:interferon regulatory factor 3;KEAP1:Kelch-like ECH-associated protein 1; KRAS: Kirsten rat sarcoma; NRF2: nuclear factor erythroid 2-like 2; NSCLC: non-small cell lung cancer; PBMCs: peripheral blood mononuclear cells; qRT-PCR: quantitative real-time polymerase chain reaction; STING: stimulator of interferon genes; 
318 TBK1:tank binding kinase 1;TILs: tumor infiltrating lymphocytes; TIME: tumor immune

319 microenvironment.

320 Acknowledgments

321 We would like to thank Editage (https://www.editage.cn) for English language editing.

322 Authors' contributions

323 CY and SX designed and supervised the project. SX, ZP, and LY carried out the experiments and 324 drafted the manuscript. LH and SX performed statistical analysis and finalized the manuscript. All 325 authors read and approved the final manuscript.

326 Funding

327 This work was supported by the National Natural Science Foundation of China (grant number 328 82103343); Scientific Research Project of Jilin Provincial Health and Family Planning Commission 329 (grant number 2018J021); Science and Technology Agency of Jilin Provincial Project (grant number 330 202002063JC) for financial support.

331

332 333

\section{Availability of data and materials}

The data sets used and/or analyzed during the current study are available from the corresponding author on reasonable request.

\section{Declarations}

\section{Ethics approval and consent to participate}

Not applicable.

\section{Consent for publication}

All authors reviewed and approved the fnal manuscript. All authors supported publication of this manuscript.

\section{Competing interests}

The authors declare that they have no competing interests. 


\section{References}

1. Bray F, Ferlay J, Soerjomataram I, Siegel RL, Torre LA, Jemal A. Global cancer statistics 2018: GLOBOCAN estimates of incidence and mortality worldwide for 36 cancers in 185 countries. CA Cancer J Clin 2018;68:394-424.

2. Sun S, Schiller JH, Gazdar AF. Lung cancer in never smokers--a different disease. Nat Rev Cancer 2007;7:778-90.

3. Drosten M, Barbacid M. Targeting the MAPK Pathway in KRAS-Driven Tumors. Cancer Cell 2020;37:543-50.

4. Gadgeel S, Rodriguez-Abreu D, Felip E, Esteban E, Speranza G, Reck M, Hui R, Boyer M, Garon EB, Horinouchi H, Cristescu R, Aurora-Garg D, et al. LBA5 - KRAS mutational status and efficacy in KEYNOTE-189: Pembrolizumab (pembro) plus chemotherapy (chemo) vs placebo plus chemo as first-line therapy for metastatic non-squamous NSCLC. Ann Oncol 2019;30:64-5.

5. Herbst RS, Lopes G, Kowalski DM, Kasahara K, Wu Y-L, De Castro G, Cho BC, Turna HZ, Cristescu R, Aurora-Garg D, Lunceford J, Kobie J, et al. LBA4 Association of KRAS mutational status with response to pembrolizumab monotherapy given as first-line therapy for PD-L1-positive advanced non-squamous NSCLC in Keynote-042. Ann Oncol 2019;30:xi63-4.

6. Borghaei H, Paz-Ares L, Horn L, Spigel DR, Steins M, Ready NE, Chow LQ, Vokes EE, Felip E, Holgado E, Barlesi F, Kohlhäufl $\mathrm{M}$, et al. Nivolumab versus Docetaxel in Advanced Nonsquamous Non-Small-Cell Lung Cancer. N Engl J Med 2015;373:1627-39.

7. Skoulidis F, Heymach J V. Co-occurring genomic alterations in non-small-cell lung cancer biology and therapy. Nat Rev Cancer 2019;19:495-509.

8. Cai MC, Chen M, Ma P, Wu J, Lu H, Zhang S, Liu J, Zhao X, Zhuang G, Yu Z, Fu Y. Clinicopathological, microenvironmental and genetic determinants of molecular subtypes in KEAP1/NRF2-mutant lung cancer. Int J Cancer 2019;144:788-801.

9. Cristescu R, Mogg R, Ayers M, Albright A, Murphy E, Yearley J, Sher X, Liu XQ, Lu H, Nebozhyn M, Zhang C, Lunceford JK, et al. Pan-tumor genomic biomarkers for PD-1 checkpoint blockade-based immunotherapy. Science (80- ) 2018;362.

10. Best SA, De Souza DP, Kersbergen A, Policheni AN, Dayalan S, Tull D, Rathi V, Gray DH, Ritchie ME, McConville MJ, Sutherland KD. Synergy between the KEAP1/NRF2 and PI3K Pathways Drives Non-Small-Cell Lung Cancer with an Altered Immune Microenvironment. Cell Metab 2018;27:935-43.

11. Camus M, Tosolini M, Mlecnik B, Pagès F, Kirilovsky A, Berger A, Costes A, Bindea G, Charoentong P, Bruneval P, Trajanoski Z, Fridman W-H, et al. Coordination of intratumoral immune reaction and human colorectal cancer recurrence. Cancer Res 2009;69:2685-93.

12. Kato D, Yaguchi T, Iwata T, Morii K, Nakagawa T, Nishimura R, Kawakami Y. Prospects for personalized combination immunotherapy for solid tumors based on adoptive cell therapies 
and immune checkpoint blockade therapies. Nihon Rinsho Meneki Gakkai Kaishi 2017;40:6877.

13. Spranger S, Bao R, Gajewski TF. Melanoma-intrinsic $\beta$-catenin signalling prevents antitumour immunity. Nature 2015;523:231-5.

14. Li A, Yi M, Qin S, Song Y, Chu Q, Wu K. Activating cGAS-STING pathway for the optimal effect of cancer immunotherapy. J Hematol Oncol 2019;12:35.

15. Della Corte CM, Sen T, Gay CM, Ramkumar K, Diao L, Cardnell RJ, Rodriguez BL, Stewart CA, Papadimitrakopoulou VA, Gibson L, Fradette JJ, Wang Q, et al. STING Pathway Expression Identifies NSCLC With an Immune-Responsive Phenotype. J Thorac Oncol 2020;15:777-91.

16. Kitajima S, Ivanova E, Guo S, Yoshida R, Campisi M, Sundararaman SK, Tange S, Mitsuishi Y, Thai TC, Masuda S, Piel BP, Sholl LM, et al. Suppression of STING Associated with LKB1 Loss in KRAS-Driven Lung Cancer. Cancer Discov 2019;9:34-45.

17. Hellyer JA, Padda SK, Diehn M, Wakelee HA. Clinical Implications of KEAP1-NFE2L2 Mutations in NSCLC. J Thorac Oncol 2021;16:395-403.

18. Olagnier D, Brandtoft AM, Gunderstofte C, Villadsen NL, Krapp C, Thielke AL, Laustsen A, Peri S, Hansen AL, Bonefeld L, Thyrsted J, Bruun V, et al. Nrf2 negatively regulates STING indicating a link between antiviral sensing and metabolic reprogramming. Nat Commun 2018;9:3506.

19. Do HTT, Lee CH, Cho J. Chemokines and their Receptors: Multifaceted Roles in Cancer Progression and Potential Value as Cancer Prognostic Markers. Cancers (Basel) 2020;12:287.

20. Parkes EE, Walker SM, Taggart LE, McCabe N, Knight LA, Wilkinson R, McCloskey KD, Buckley NE, Savage KI, Salto-Tellez M, McQuaid S, Harte MT, et al. Activation of STINGDependent Innate Immune Signaling By S-Phase-Specific DNA Damage in Breast Cancer. J Natl Cancer Inst 2017;109:djw199.

21. Sen T, Rodriguez BL, Chen L, Corte CM Della, Morikawa N, Fujimoto J, Cristea S, Nguyen T, Diao L, Li L, Fan Y, Yang Y, et al. Targeting DNA Damage Response Promotes Antitumor Immunity through STING-Mediated T-cell Activation in Small Cell Lung Cancer. Cancer Discov 2019;9:646-61.

22. Kwon J, Bakhoum SF. The Cytosolic DNA-Sensing cGAS-STING Pathway in Cancer. Cancer Discov 2020;10:26-39.

23. Gong W, Lu L, Zhou Y, Liu J, Ma H, Fu L, Huang S, Zhang Y, Zhang A, Jia Z. The novel STING antagonist H151 ameliorates cisplatin-induced acute kidney injury and mitochondrial dysfunction. Am J Physiol Ren Physiol 2021;320:F608-16.

24. Haag SM, Gulen MF, Reymond L, Gibelin A, Abrami L, Decout A, Heymann M, van der Goot FG, Turcatti G, Behrendt R, Ablasser A. Targeting STING with covalent small-molecule inhibitors. Nature 2018;559:269-73. 
25. Ferrer I, Zugazagoitia J, Herbertz S, John W, Paz-Ares L, Schmid-Bindert G. KRAS-Mutant non-small cell lung cancer: From biology to therapy. Lung Cancer 2018;124:53-64.

26. Dong Z-Y, Zhong W-Z, Zhang X-C, Su J, Xie Z, Liu S-Y, Tu H-Y, Chen H-J, Sun Y-L, Zhou Q, Yang J-J, Yang X-N, et al. Potential Predictive Value of TP53 and KRAS Mutation Status for Response to PD-1 Blockade Immunotherapy in Lung Adenocarcinoma. Clin Cancer Res 2017;23:3012-24.

27. Takashima K, Takeda Y, Oshiumi H, Shime H, Okabe M, Ikawa M, Matsumoto M, Seya T. STING in tumor and host cells cooperatively work for NK cell-mediated tumor growth retardation. Biochem Biophys Res Commun 2016;478:1764-71.

28. Ho SSW, Zhang WYL, Tan NYJ, Khatoo M, Suter MA, Tripathi S, Cheung FSG, Lim WK, Tan PH, Ngeow J, Gasser S. The DNA Structure-Specific Endonuclease MUS81 Mediates DNA Sensor STING-Dependent Host Rejection of Prostate Cancer Cells. Immunity 2016;44:1177-89.

29. Zhou L, Xu Q, Huang L, Jin J, Zuo X, Zhang Q, Ye L, Zhu S, Zhan P, Ren J, Lv T, Song Y. Low-dose carboplatin reprograms tumor immune microenvironment through STING signaling pathway and synergizes with PD-1 inhibitors in lung cancer. Cancer Lett 2021;500:163-71.

\section{Figure legends}

FIGURE 1 NRF2 was overexpressed and activated in KRAS-KEAP1 co-mutation cells. (A) The mutation characters of cell lines used in this study were identified through CCLE database. (B) The protein and (C) mRNA expression of NRF2 were determined by western blot and qRT-PCR, respectively. (D) Nuclear and cytoplasm isolation assay and (E) Immunofluorescence staining was performed to detect NRF2 localization in both KK and K type NSCLC cell lines (10×). Scale bar = $100 \mu \mathrm{m}$. Data are shown as mean $\pm \mathrm{SD}$ of three independent experiments.

FIGURE 2 NRF2 negatively regulated $\mathrm{CD} 8^{+} \mathrm{T}$ cells recruitment and its chemokines. (A) The expression of NRF2 was negatively correlated with $\mathrm{CD} 8^{+} \mathrm{T}$ cell abundance based on TISIDB database. GEPIA database was used to observe the relativity between NRF2 and chemokines (B) CCL5, and (C) CXCL10 in lung adenocarcinoma (LUAD). (D, E) NRF2 was upregulated in K type cells and $(\mathbf{F}, \mathbf{G})$ knocked down in $\mathrm{KK}$ type cells. $\mathrm{CD}^{+} / \mathrm{CD}^{+} \mathrm{T}$ cells recruitment was evaluated by the chemotaxis assay; cells that migrated to the lower chamber were stained and recognized by a flow cytometer, and analyzed by Flowjo software. The mRNA levels of CCL5 and CXCL10 was detected after (H) NRF2 overexpressed in K type cells and (I) downregulated in KK type cells by qRT-PCR. ELISA was performed to determine the secreted levels of CCL5 and CXCL10 after (J) upregulating NRF2 in K type cells and (K) downregulating NRF2 in KK type cells. EV, empty vector; NC, 
negative control; si-NRF2, small interfere RNA that target NRF2. Data are shown as mean \pm SD of at least three independent experiments. $* P<0.05$, $* * P<0.01, * * * P<0.001$.

FIGURE 3 STING effected as a tumor suppressor in NSCLC. (A) The mRNA levels of STING in a number of human tumors and corresponding normal tissues were observed through GEPIA database, including (B) lung adenocarcinoma (LUAD). (C) The protein expression of STING in LUAD and normal tissues were confirmed by immunohistochemistry results from the HPA database. Scale bar = $50 \mu \mathrm{m}$. (D) The protein expression of STING in normal bronchial epithelial HBE cells and two NSCLC cell lines were determined by western blot and the relative gray values were shown in the histogram. The results from the Kaplan-Meier Plotter database of patients (E) overall survival (OS) and (F) progress free survival (PFS) with relatively high or low STING expression. Data are shown as mean \pm SD. $* P<0.05, * * P<0.01$.

FIGURE 4 STING pathway was negatively regulated by NRF2. (A) The relationship between STING and NRF2 was analyzed by GEPIA database. The expression of STING in both KK and K type cells were determined by (B) western blot and (C) qRT-PCR. (D) The interfere efficiency of three specific NRF2 target siRNA sequences were detected in A549 and H460 cells by western blot. No.3 siRNA sequence exerted the highest transfection efficiency and was highlighted by red dotted frames. (E) Changes of key protein levels in STING pathway were tested by western blotting after up- or down-regulating NRF2 in K and KK type cells, respectively. (F) The relative western blot gray values were shown in the histogram. EV, empty vector; NC, negative control; si-NRF2, small interfere RNA that target NRF2. Data are shown as mean $\pm \mathrm{SD}$ of three independent experiments. ${ }^{*} P$ $<0.05, * * P<0.01$.

FIGURE 5 NRF2-mediated suppressive TIME of KK type NSCLC was dependent on inhibiting STING pathway. (A) The inhibitory efficacy of H151 on STING pathway in A549 and H460 cells transfected with NRF2 siRNA were tested by western blot. The activity of STING pathway was significant inhibited after exposure to $0.5 \mu \mathrm{M} \mathrm{H} 151$ for $3 \mathrm{~h}$ and was highlighted by red dotted frames. After knocked down NRF2 and the addition of H151 in A549 and H460 cells, (B) the mRNA levels and (C) the secreted levels of CCL5 and CXCL10 were tested by qRT-PCR and ELISA, respectively. (D) $\mathrm{CD}^{+} / \mathrm{CD}^{+} \mathrm{T}$ cells recruitment was evaluated by the chemotaxis assay; cells that migrated to the lower chamber were stained and recognized by a flow cytometer, and analyzed by Flowjo software. $\mathrm{NC}$, negative control; si-NRF2, small interfere RNA that target NRF2. Data are shown as mean $\pm \mathrm{SD}$ of three independent experiments. $* P<0.05, * * P<0.01$. 

FIGURE 6 NRF2 promoted BRCA1 expression and DNA damage repair to inhibit STING pathway. (A) The functional associations between target proteins was predicted using String database. (B) The correlations between BRCA1 and STING or NRF2 were analyzed by GEPIA database. (C) The expression of BRCA1 in both KK and K type cells were determined by western blot. (D) Changes of regulating NRF2 in K and KK type cells, respectively. The relative western blot gray values were shown in the histogram. (E) Binding sites between the BRCA1 promoter sequence and NRF2 were predicted by JASPAR database. (F) Luciferase activities following transfection with NRF2 expression vector and pGL3-Basic or BRCA1 vector were detected by dual luciferase reporter assay. pRL-TK vectors were transfected as internal control. (G) The interfere efficiency of three specific BRCA1 target siRNA sequences were detected in A549 and H460 cells by western blot. No.1 siRNA sequence exerted the highest transfection efficiency and was highlighted by red dotted frames. $(\mathbf{H})$ Changes of BRCA1, NRF2 and key protein levels in STING pathway were tested by western blot after down-regulating BRCA1 in A549 and H460 cells. The relative western blot gray values were shown in the histogram. EV, empty vector; NC, negative control; si-NRF2, small interfere RNA that target NRF2. si-B, small interfere RNA that target BRCA1. Data are shown as mean \pm SD of three independent experiments. ${ }^{*} P<0.05, * * P<0.01$, ns: not significant.

FIGURE 7 Schematic representation of the potential mechanisms of "hot" and "cold" tumors in K and KK type NSCLC. (A) In K type cells, exogenous risks lead to DNA damage, increase the cytoplasmic double-stranded DNA (dsDNA), and activate the STING pathway, resulting in the recruitment of $\mathrm{CD}^{+} \mathrm{T}$ cells by promoting the chemokines CCL5 and CXCL10, leading to a "hot tumor" ; (B) In KK type cells, when KEAP1 mutation (KEAP1 MUT) occurred, NRF2 translocated into nuclear and bond to the ARE sequence in the promoter region of the BRCAl gene, to promote BRCA1 transcription and expression, and DNA damage repair, resulting in STING pathway inactivation, chemokines synthesis and secretion reduction, and $\mathrm{CD}^{+} \mathrm{T}$ cell recruitment inhibition, leading to a suppressive TIME (cold tumor). 


\section{Figures}

A

\begin{tabular}{|c|c|c|c|c|}
\hline Cell line & Mutation gene & Variant classification & Variant Type & Protein Change \\
\hline \multirow{2}{*}{ A549 } & $\begin{array}{l}\text { KRAS } \\
\text { KEAP1 }\end{array}$ & Missense Mutation & SNP & p.G12S \\
\hline H460 & $\begin{array}{l}\text { KRAS } \\
\text { KEAP1 }\end{array}$ & Missense Mutation & SNP & p.G333C \\
\hline CALU-1 & KRAS & Missense Mutation & SNP & p.Q61H \\
\hline H358 & KRAS & Missense Mutation & SNP & p.D236H \\
\hline
\end{tabular}

B

$\mathrm{C}$
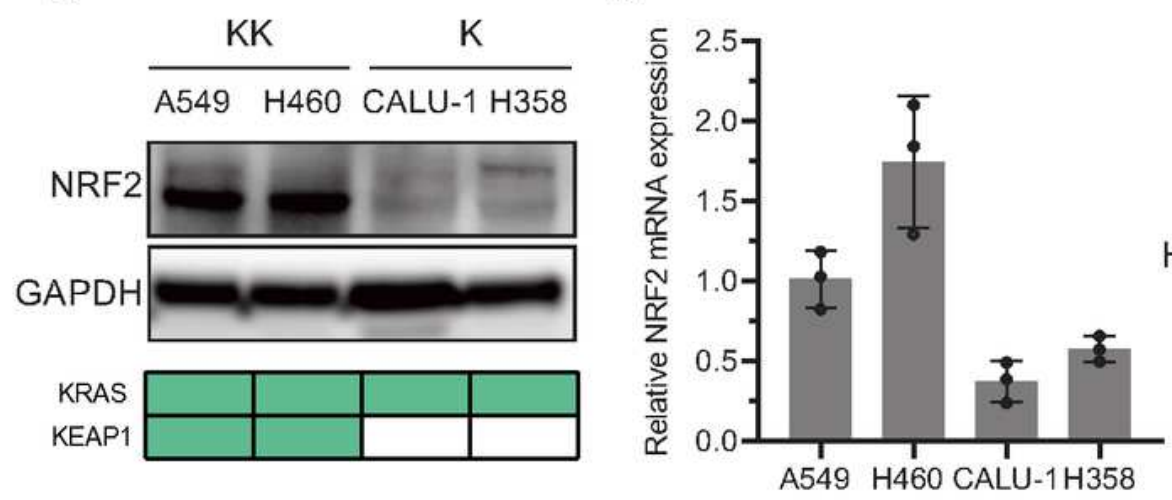

D
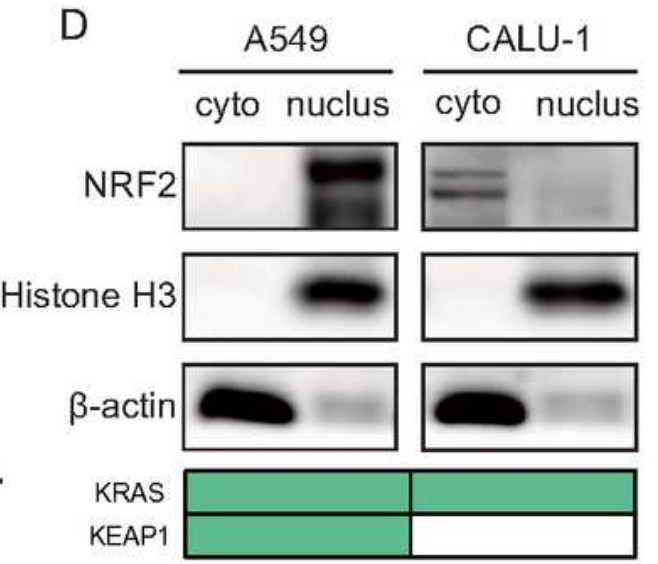

$\mathrm{E}$

DAPI

FITC

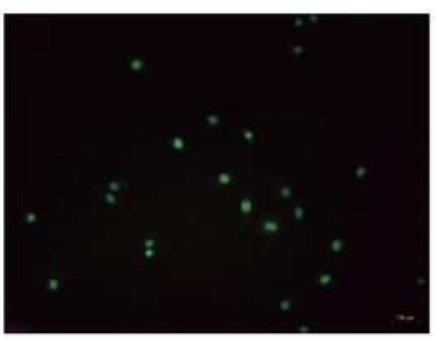

MERGE

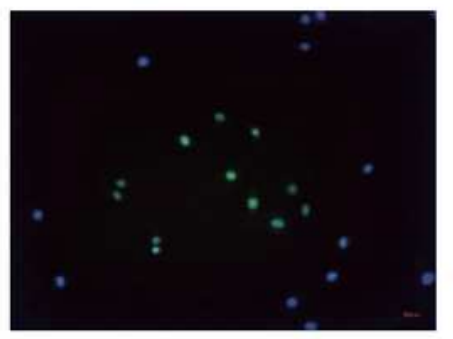

CALU-1
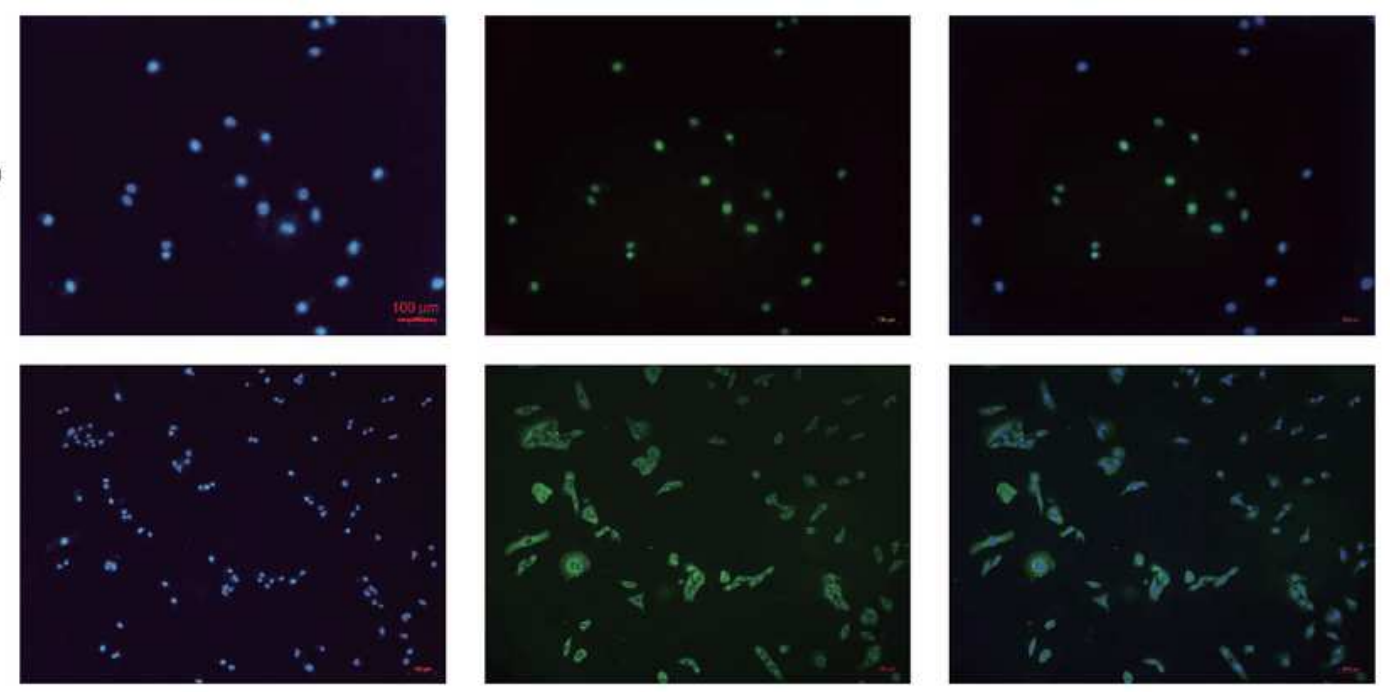

\section{Figure 1}

NRF2 was overexpressed and activated in KRAS-KEAP1 co-mutation cells. (A) The mutation characters of cell lines used in this study were identified through CCLE database. (B) The protein and (C) mRNA expression of NRF2 were determined by western blot and qRT-PCR, respectively. (D) Nuclear and 
cytoplasm isolation assay and (E) Immunofluorescence staining was performed to detect NRF2 localization in both KK and K type NSCLC cell lines (10x). Scale bar $=100 \mu \mathrm{m}$. Data are shown as mean \pm SD of three independent experiments.
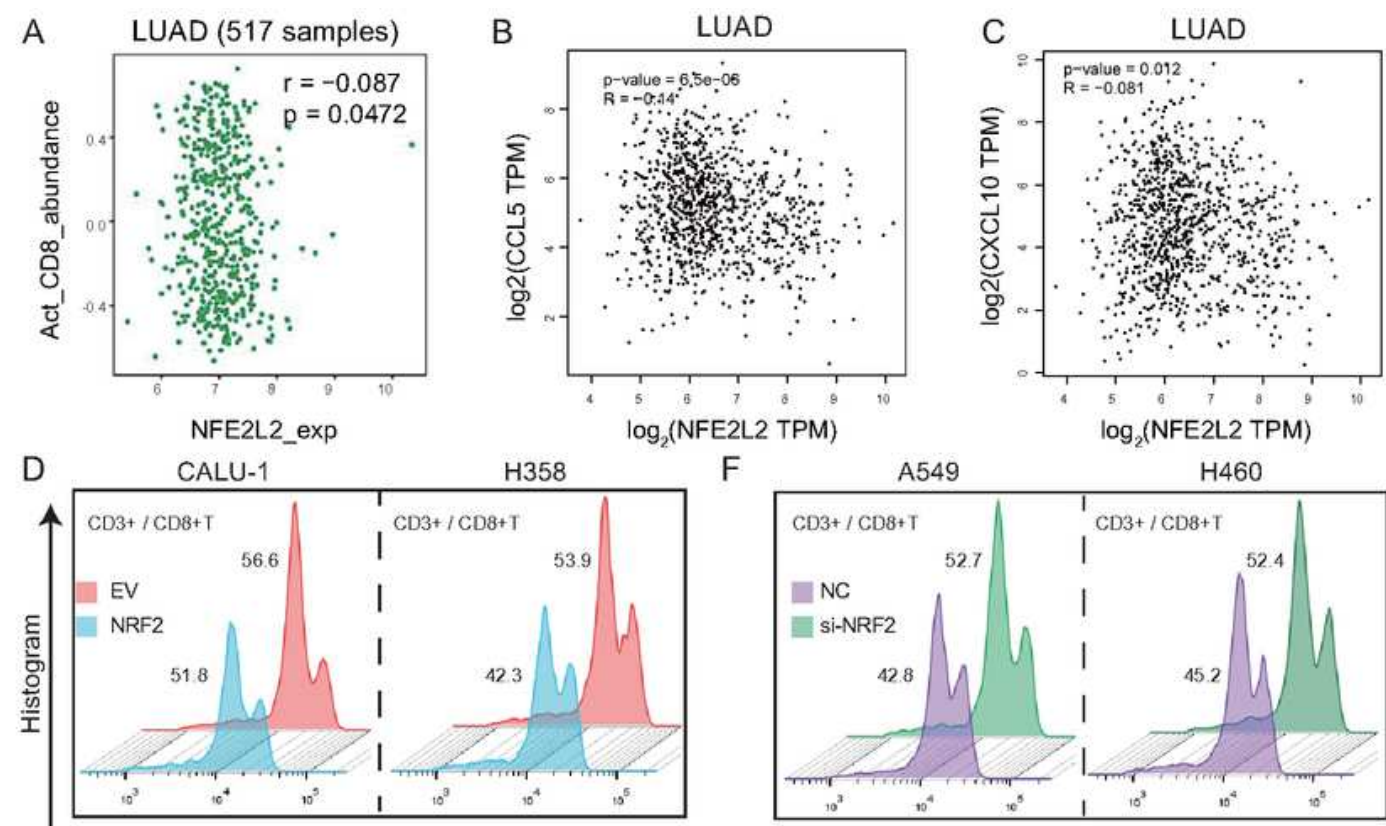

$\mathrm{F}$

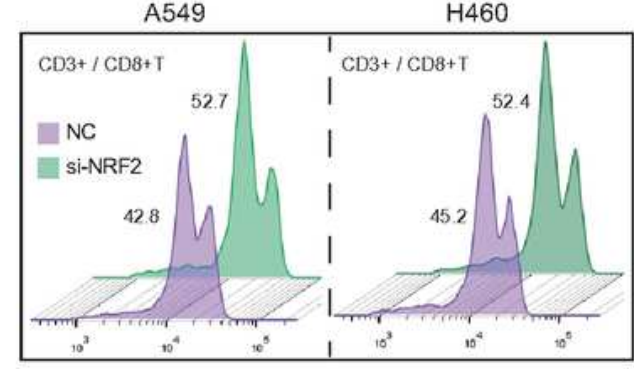

$\mathrm{E}$

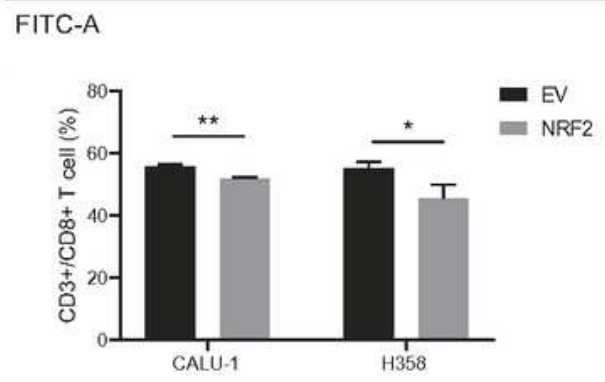

H

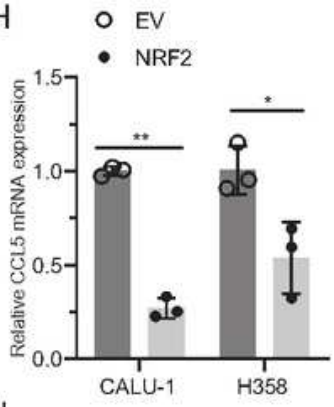

○ $\mathrm{EV}$

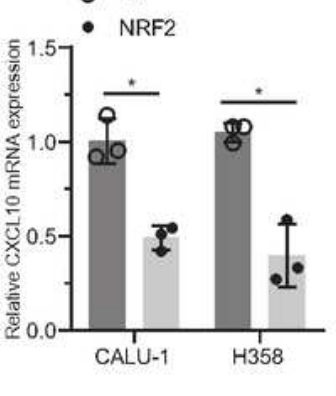

G
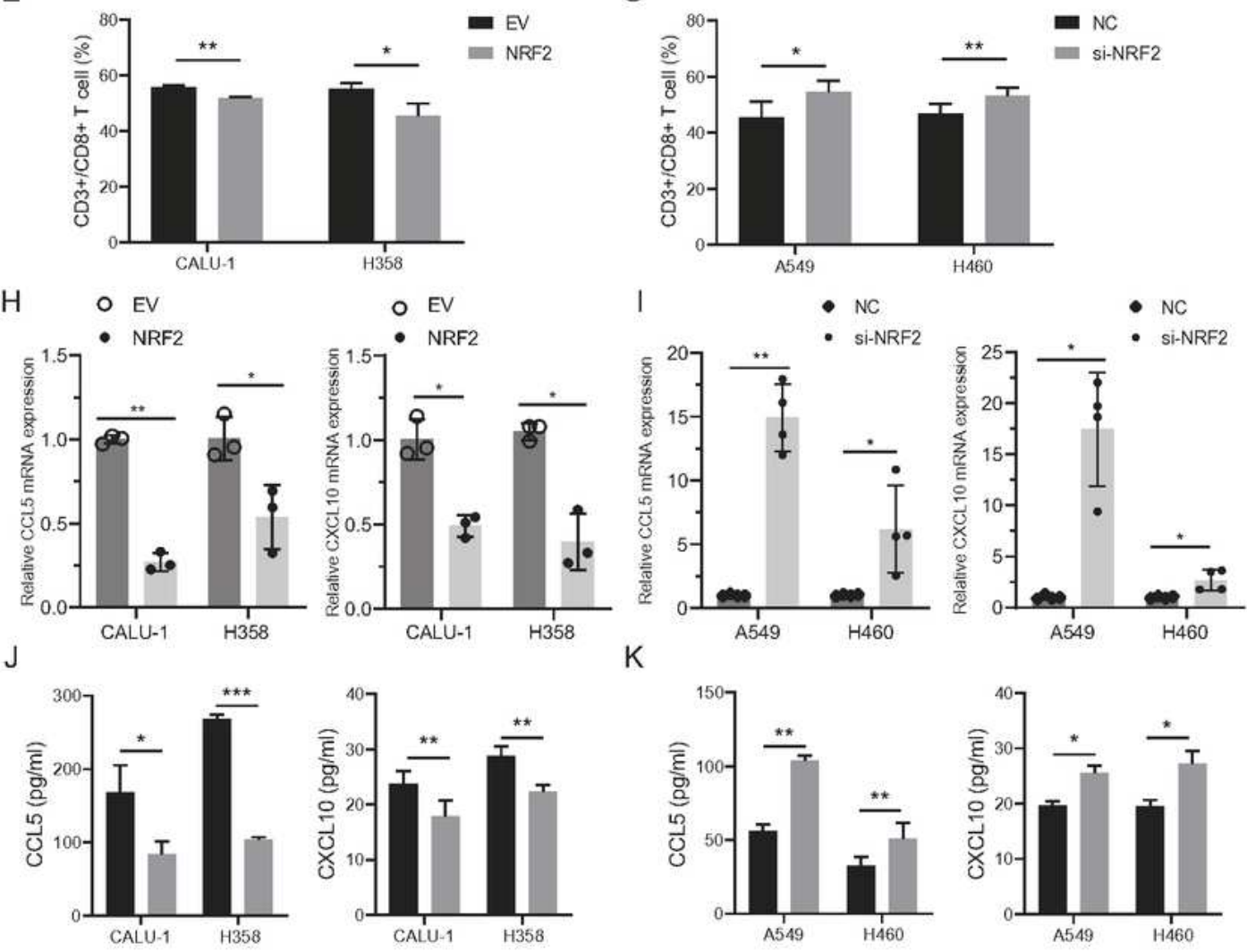

Figure 2 
NRF2 negatively regulated $C D 8^{+} T$ cells recruitment and its chemokines. (A) The expression of NRF2 was negatively correlated with CD8+ T cell abundance based on TISIDB database. GEPIA database was used to observe the relativity between NRF2 and chemokines (B) CCL5, and (C) CXCL10 in lung adenocarcinoma (LUAD). (D, E) NRF2 was upregulated in K type cells and $(F, G)$ knocked down in KK type cells. $\mathrm{CD}^{+} / \mathrm{CD}^{+} \mathrm{T}$ cells recruitment was evaluated by the chemotaxis assay; cells that migrated to the lower chamber were stained and recognized by a flow cytometer, and analyzed by Flowjo software. The mRNA levels of CCL5 and CXCL10 was detected after $(\mathrm{H})$ NRF2 overexpressed in K type cells and (I) downregulated in KK type cells by qRT-PCR. ELISA was performed to determine the secreted levels of CCL5 and CXCL10 after ( $\mathrm{J}$ ) upregulating NRF2 in K type cells and (K) downregulating NRF2 in KK type cells. EV, empty vector; NC, 16 negative control; si-NRF2, small interfere RNA that target NRF2. Data are shown as mean $\pm S D$ of at least three independent experiments. ${ }^{*} P<0.05,{ }^{\star} P<<0.01,{ }^{\star \star \star} P<0.001$. 
A

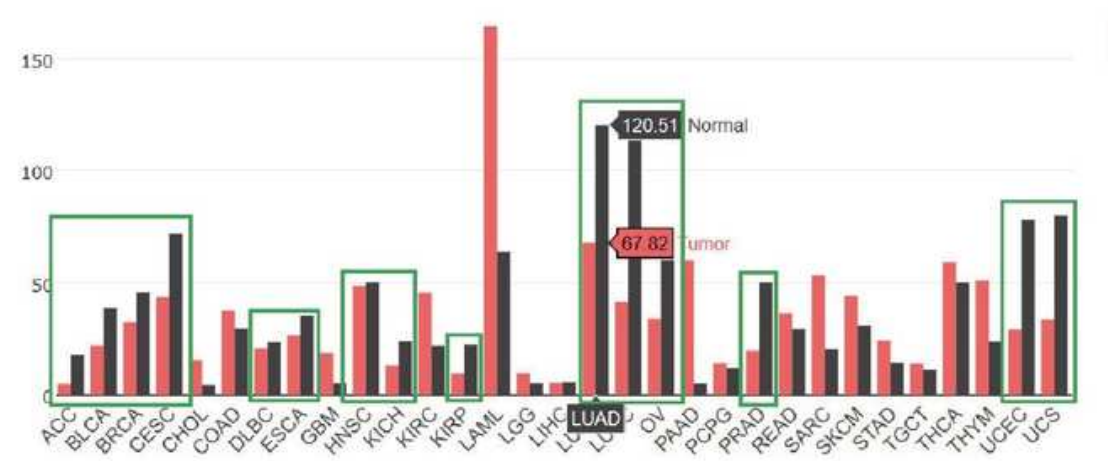

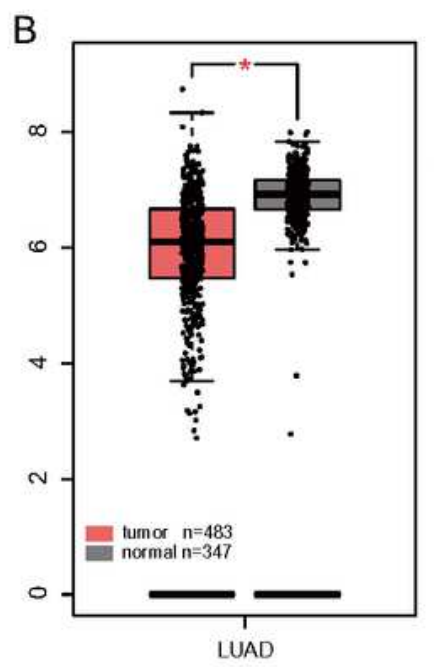

C
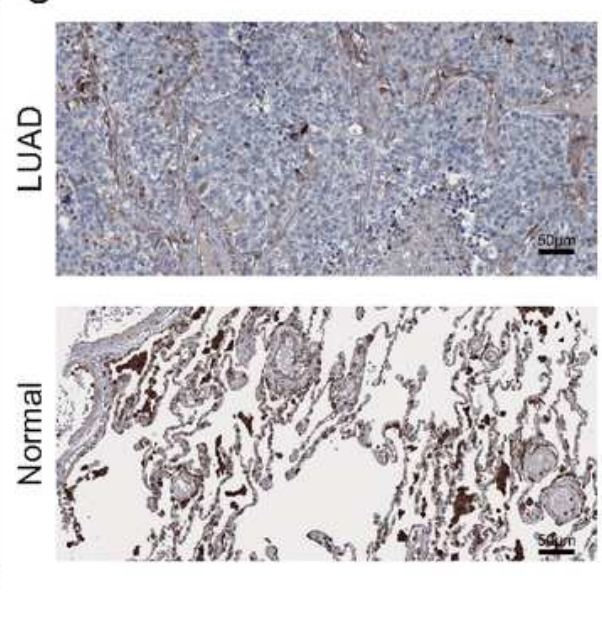

Tumor

- Normal
$\mathrm{E}$

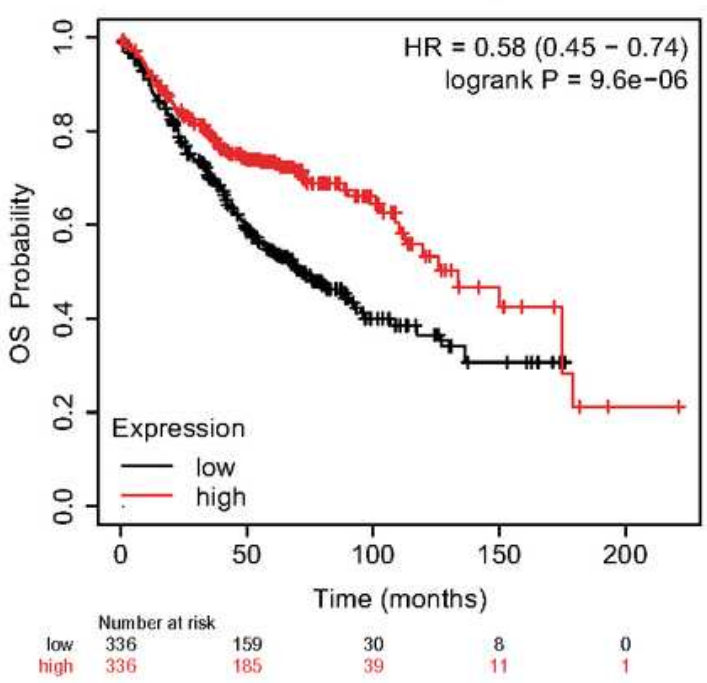

$\mathrm{F}$
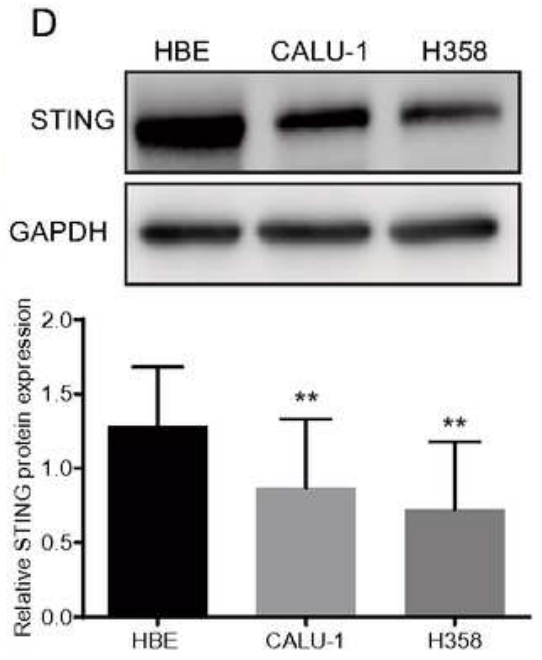

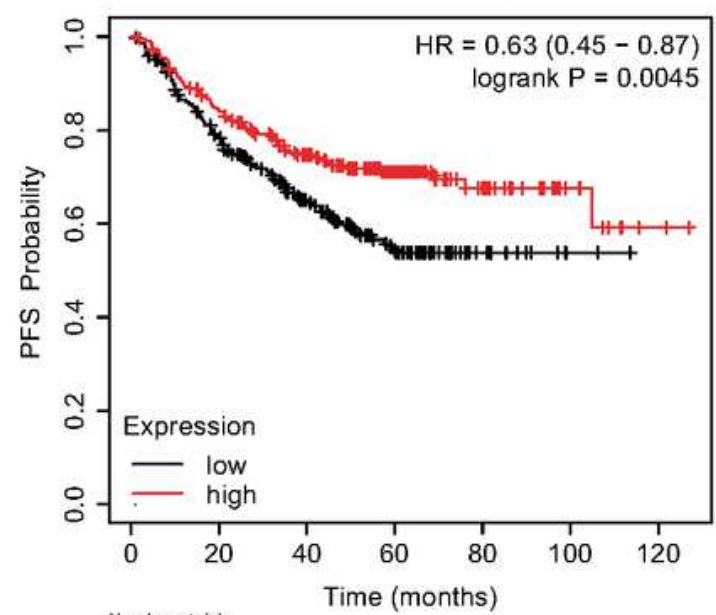

\begin{tabular}{cccccccc}
\multicolumn{7}{c}{ Number at risk } & \\
low & 222 & 160 & 104 & 58 & 12 & 2 & 0 \\
high & 221 & 181 & 140 & 91 & 32 & 11 & 2
\end{tabular}

\section{Figure 3}

STING effected as a tumor suppressor in NSCLC. (A) The mRNA levels of STING in a number of human tumors and corresponding normal tissues were observed through GEPIA database, including (B) lung adenocarcinoma (LUAD). (C) The protein expression of STING in LUAD and normal tissues were confirmed by immunohistochemistry results from the HPA database. Scale bar $=50 \mu \mathrm{m}$. (D) The protein expression of STING in normal bronchial epithelial HBE cells and two NSCLC cell lines were determined 
by western blot and the relative gray values were shown in the histogram. The results from the KaplanMeier Plotter database of patients (E) overall survival (OS) and (F) progress free survival (PFS) with relatively high or low STING expression. Data are shown 460 as mean $\pm S D$. ${ }^{*} P<0.05, * * P<0.01$.
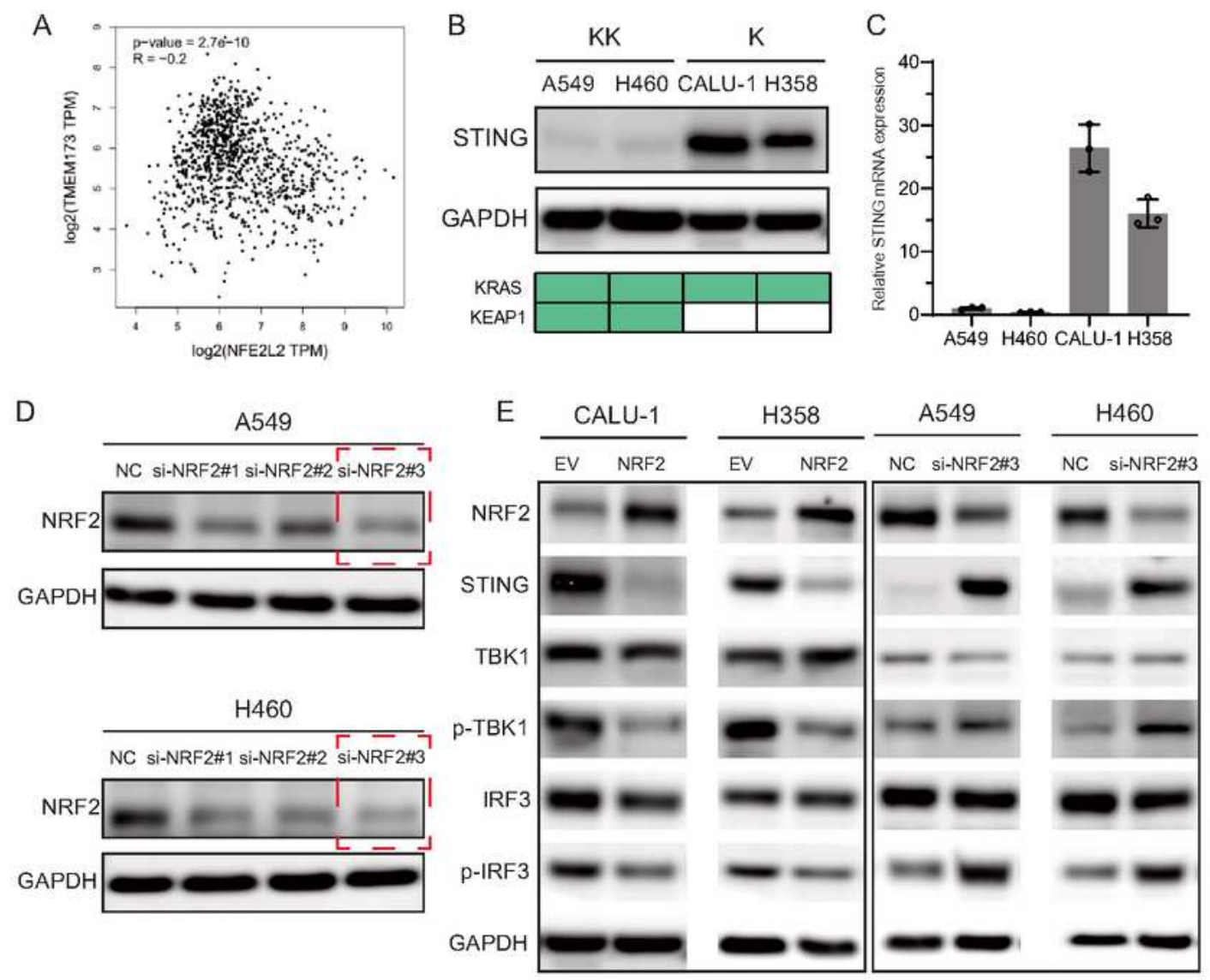

F
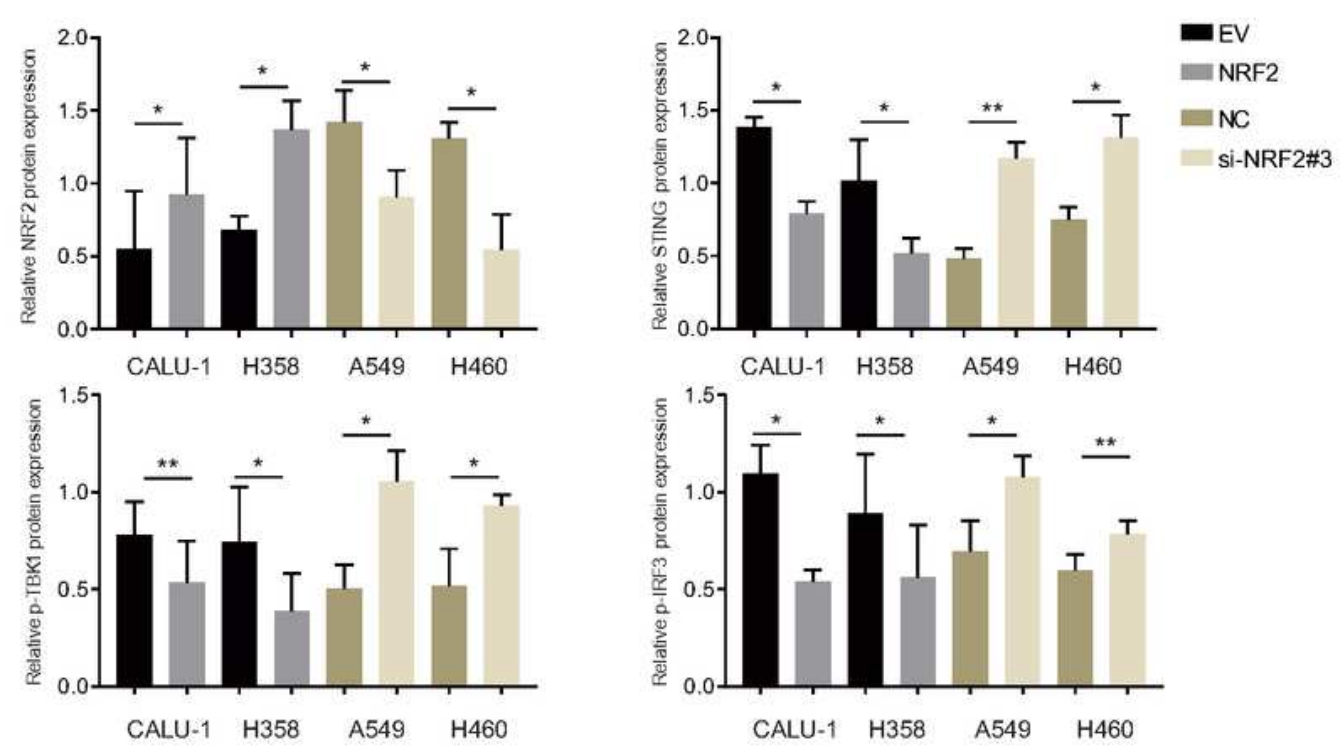

Figure 4 
STING pathway was negatively regulated by NRF2. (A) The relationship between STING and NRF2 was analyzed by GEPIA database. The expression of STING in both KK and $\mathrm{K}$ type cells were determined by (B) western blot and (C) qRT-PCR. (D) The interfere efficiency of three specific NRF2 target siRNA sequences were detected in A549 and H460 cells by western blot. No.3 siRNA sequence exerted the highest transfection efficiency and was highlighted by red dotted frames. (E) Changes of key protein levels in STING pathway were tested by western blotting after up- or down-regulating NRF2 in K and KK type cells, respectively. $(F)$ The relative western blot gray values were shown in the histogram. EV, empty vector; NC, negative control; si-NRF2, small interfere RNA that target NRF2. Data are shown as mean \pm SD of three independent experiments. ${ }^{*} \mathrm{P} 470<0.05,{ }^{*} \mathrm{P}<0.01$. 
A

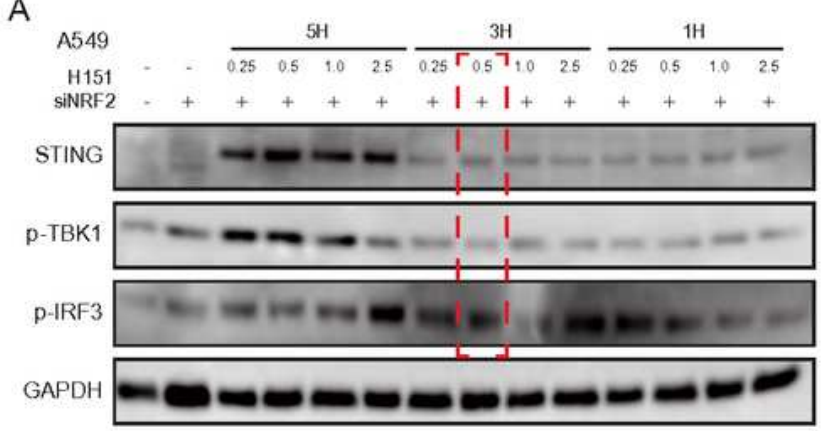

B
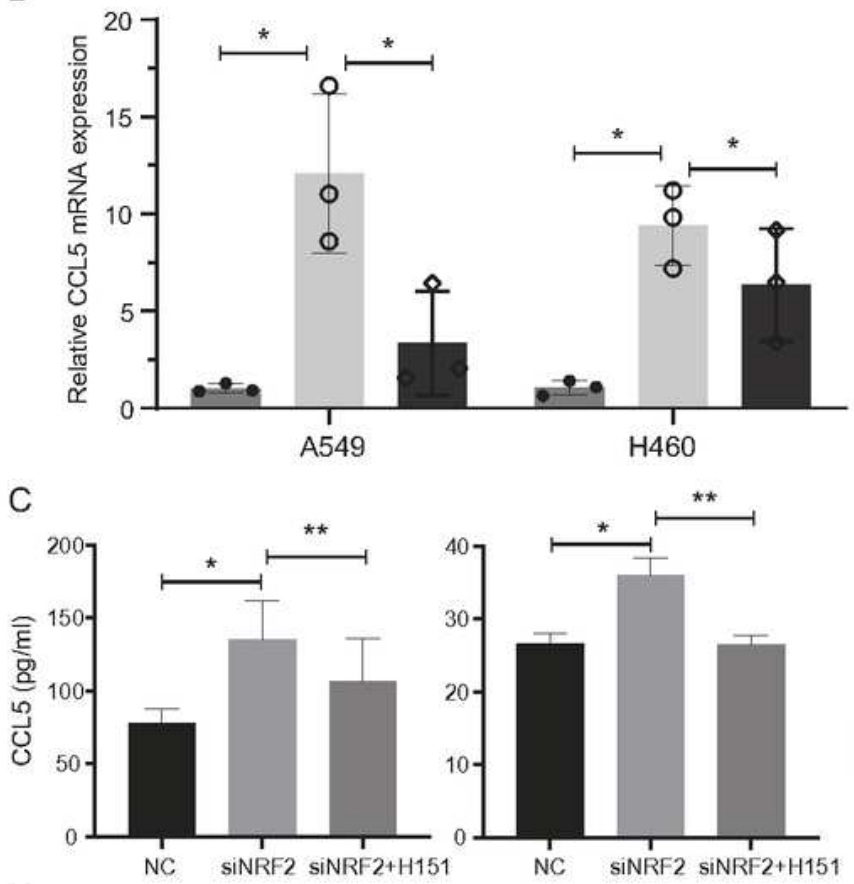

D
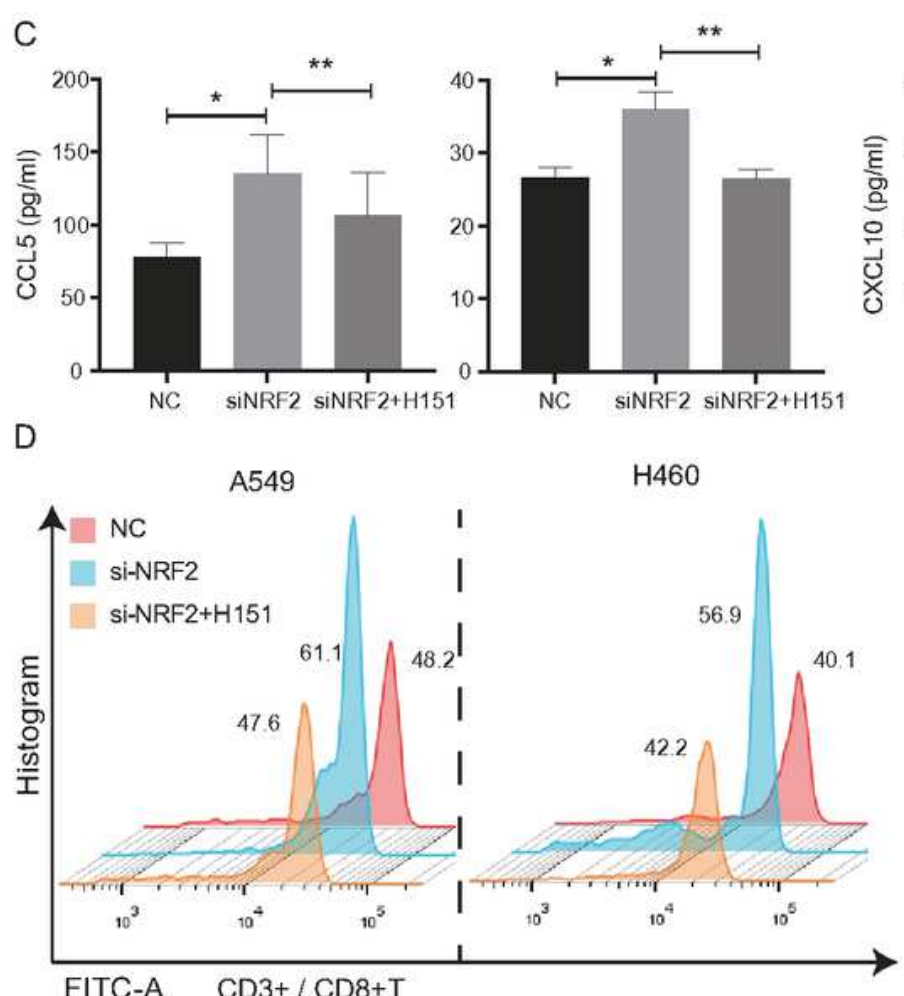

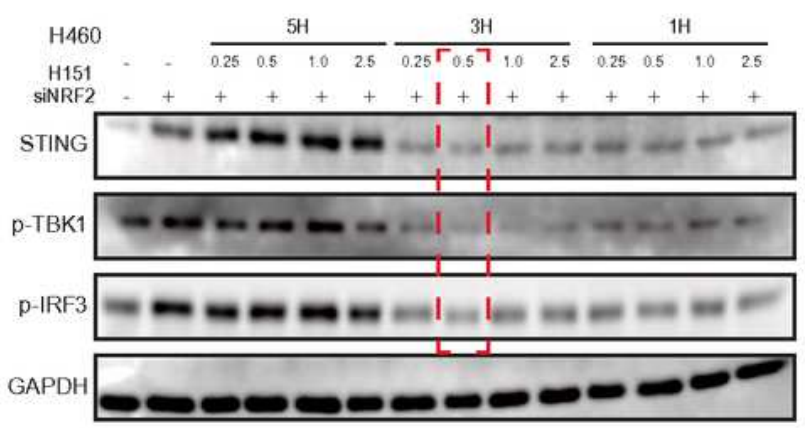

- NC

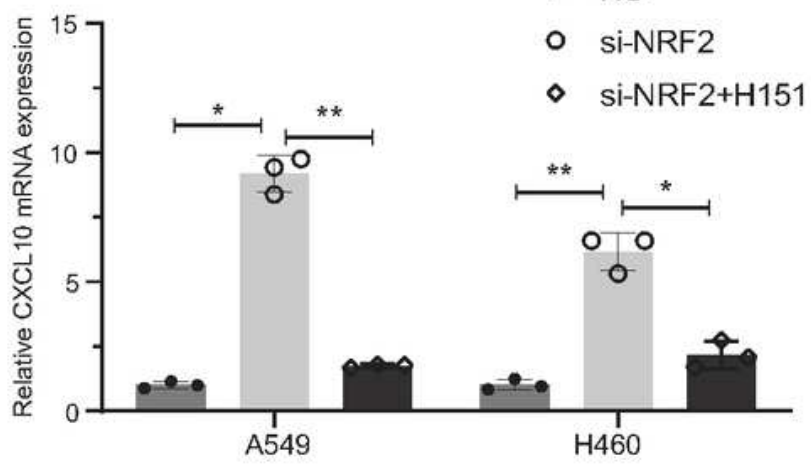

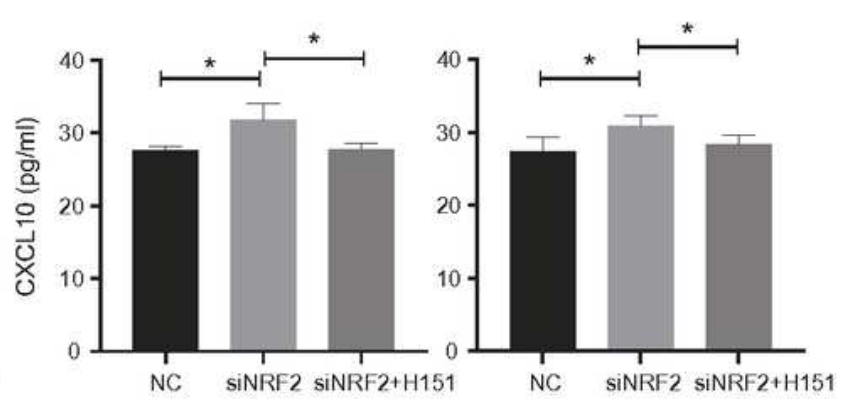

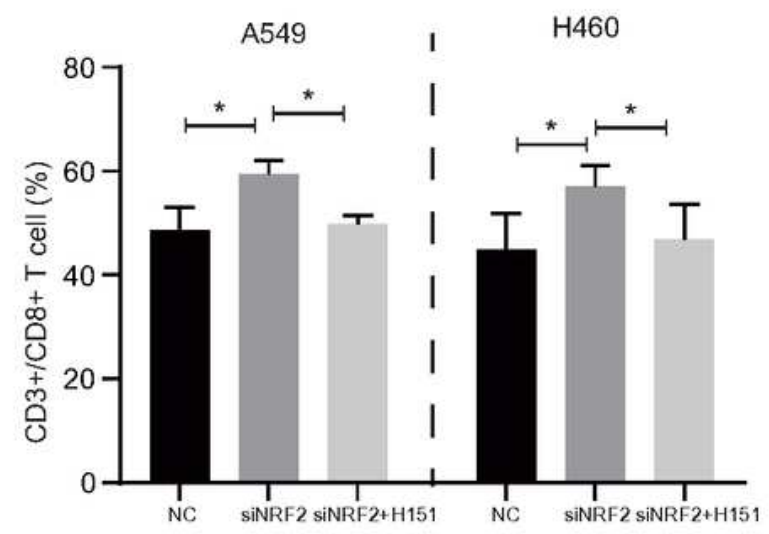

\section{Figure 5}

NRF2-mediated suppressive TIME of KK type NSCLC was dependent on inhibiting STING pathway. (A) The inhibitory efficacy of H151 on STING pathway in A549 and H460 cells transfected with NRF2 siRNA were tested by western blot. The activity of STING pathway was significant inhibited after exposure to 0.5 $\mu \mathrm{M} \mathrm{H151}$ for $3 \mathrm{~h}$ and was highlighted by red dotted frames. After knocked down NRF2 and the addition of H151 in A549 and H460 cells, (B) the mRNA levels and (C) the secreted levels of CCL5 and CXCL10 were 
tested by qRT-PCR and ELISA, respectively. (D) $\mathrm{CD}^{+} / \mathrm{CD}^{+} \mathrm{T}$ cells recruitment was evaluated by the chemotaxis assay; cells that migrated to the lower chamber were stained and recognized by a flow cytometer, and analyzed by Flowjo software. NC, negative control; si-NRF2, small interfere RNA that target NRF2. Data are shown as mean $\pm S D$ of three independent experiments. ${ }^{*} P<0.05,{ }^{*} \mathrm{P}<0.01$.

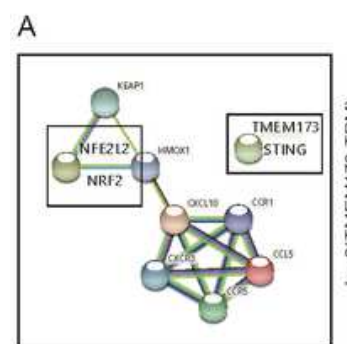

B

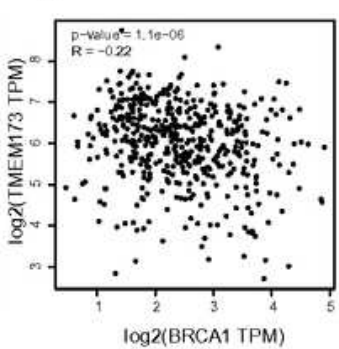

D

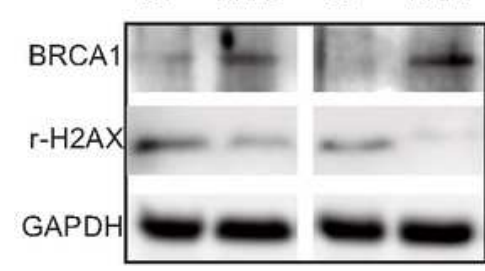

E
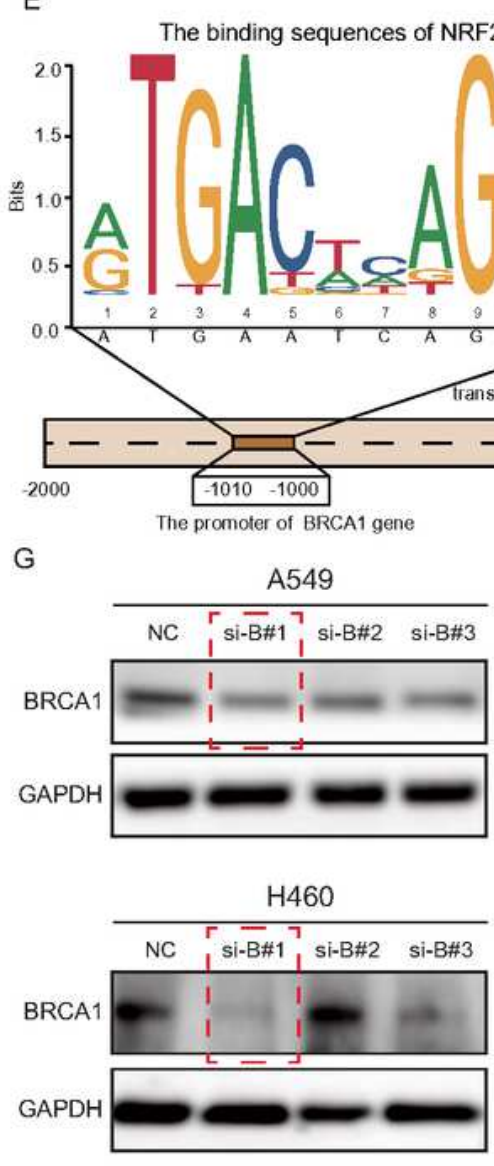

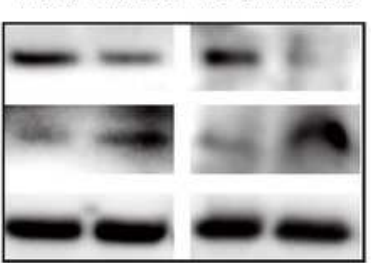

F
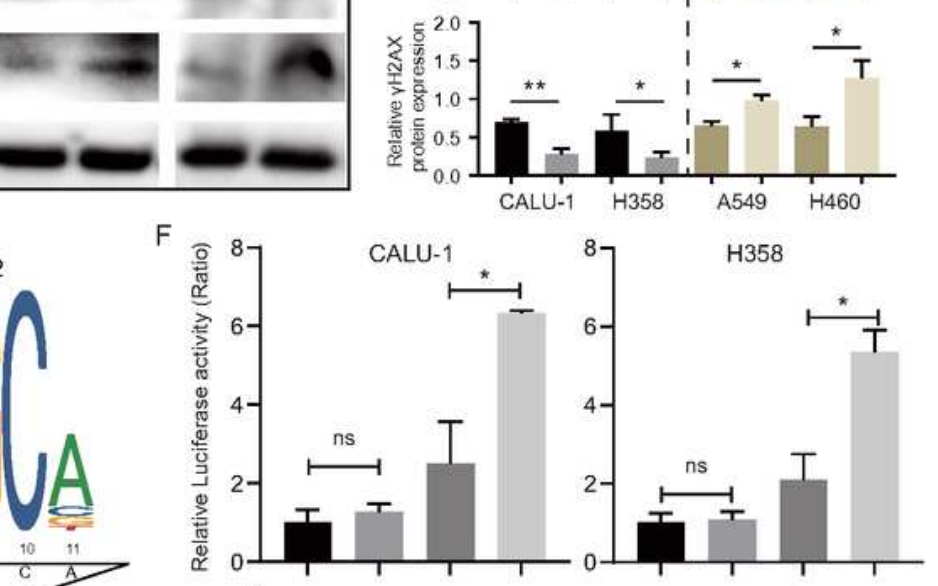

C

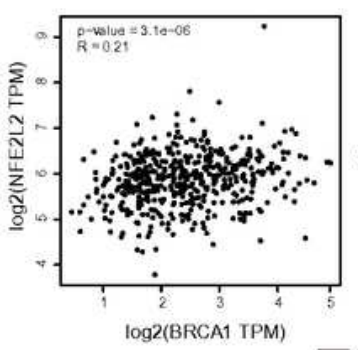

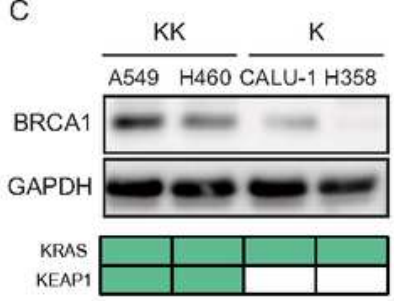

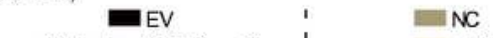

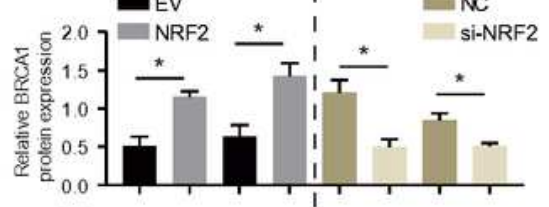
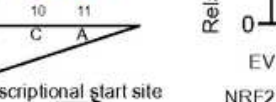

pGL3-Basic

GL3-BRCA1

$\mathrm{H}$
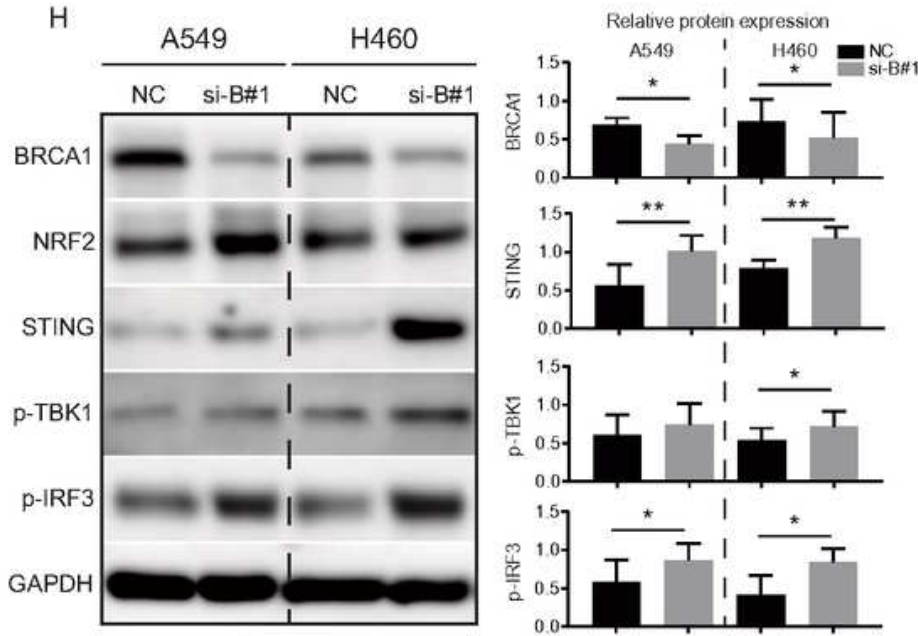

Figure 6 
NRF2 promoted BRCA1 expression and DNA damage repair to inhibit STING pathway. (A) The functional associations between target proteins was predicted using String database. (B) The correlations between BRCA1 and STING or NRF2 were analyzed by GEPIA database. (C) The expression of BRCA1 in both KK and $\mathrm{K}$ type cells were determined by western blot. (D) Changes of BRCA1 and DNA damage marker $\mathrm{Y}^{-}$ $\mathrm{H} 2 \mathrm{AX}$ levels were tested by western blot after up- or down- regulating NRF2 in K and KK type cells, respectively. The relative western blot gray values were shown in the histogram. (E) Binding sites between the BRCA1 promoter sequence and NRF2 were predicted by JASPAR database. (F) Luciferase activities following transfection with NRF2 expression vector and pGL3-Basic or BRCA1 vector were detected by dual luciferase reporter assay. pRL-TK vectors were transfected as internal control. (G) The interfere efficiency of three specific BRCA1 target siRNA sequences were detected in A549 and H460 cells by western blot. No.1 siRNA sequence exerted the highest transfection efficiency and was highlighted by red dotted frames. $(\mathrm{H})$ Changes of BRCA1, NRF2 and key protein levels in STING pathway were tested by western blot after down-regulating BRCA1 in A549 and $\mathrm{H} 460$ cells. The relative western blot gray values were 495 shown in the histogram. EV, empty vector; NC, negative control; si-NRF2, small interfere RNA that target NRF2. si-B, small interfere RNA that target BRCA1. Data are shown as mean \pm SD of three independent experiments. ${ }^{*} \mathrm{P}<0.05,{ }^{*} \mathrm{P}<0.01$, ns: not significant.

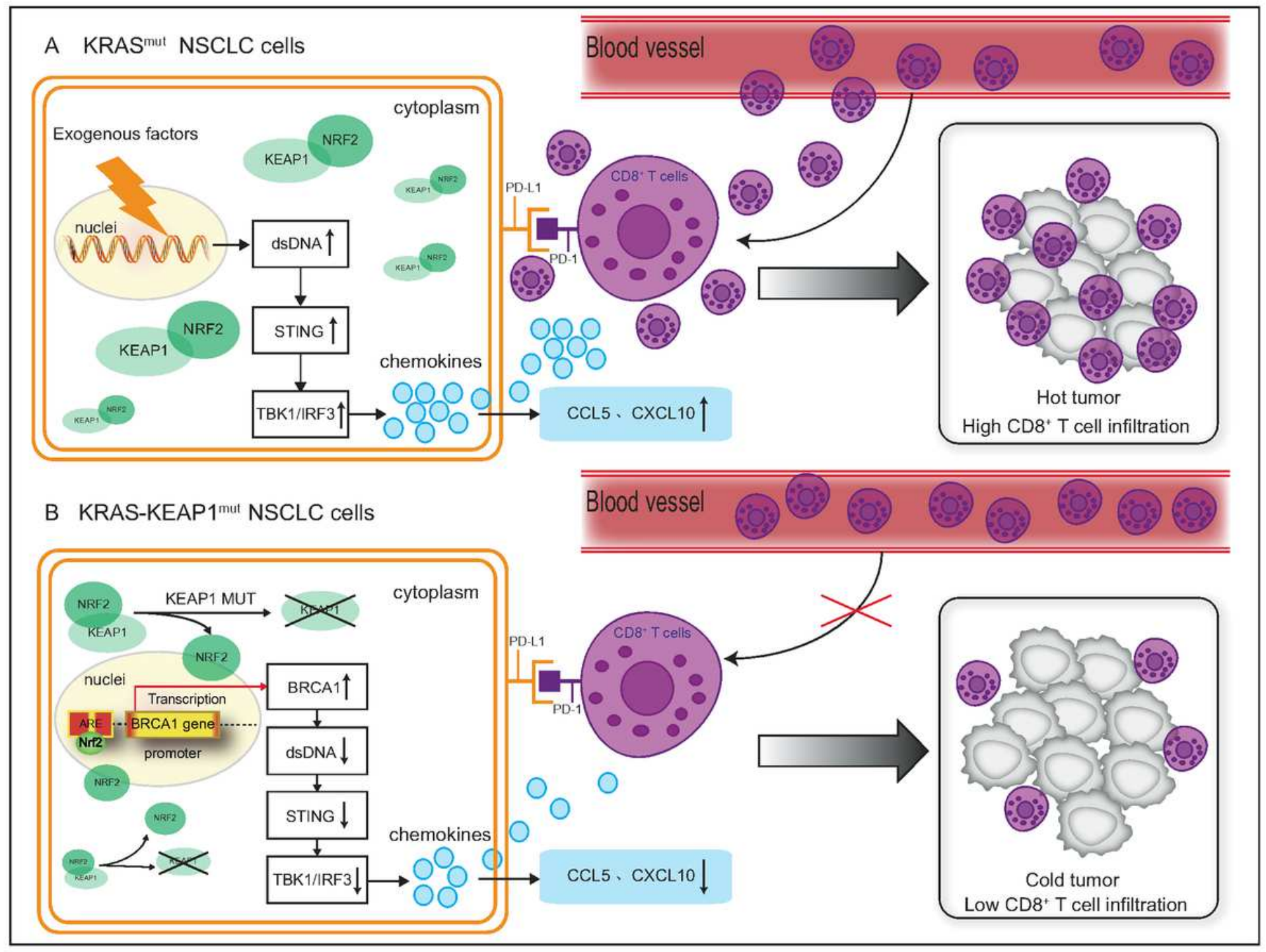




\section{Figure 7}

Schematic representation of the potential mechanisms of "hot" and "cold" tumors in K and KK type NSCLC. (A) In K type cells, exogenous risks lead to DNA damage, increase the cytoplasmic doublestranded DNA (dsDNA), and activate the STING pathway, resulting in the recruitment of $\mathrm{CD}^{+} \mathrm{T}$ cells by promoting the chemokines CCL5 and CXCL10, leading to a "hot tumor"; (B) In KK type cells, when KEAP1 mutation (KEAP1 MUT) occurred, NRF2 translocated into nuclear and bond to the ARE sequence in the promoter region of the BRCA1 gene, to promote BRCA1 transcription and expression, and DNA damage repair, resulting in STING pathway inactivation, chemokines synthesis and secretion reduction, and CD $8^{+}$ T cell recruitment inhibition, leading to a suppressive TIME (cold tumor).

\section{Supplementary Files}

This is a list of supplementary files associated with this preprint. Click to download.

- SupplementalTable3.xlsx

- SupplementalTables12.docx

- Supplementalfigurelegends.docx

- Supplentmentfigure1.pdf 